\title{
Amplitude Scaling of Active Separation Control
}

Oksana Stalnov and Avraham "Avi" Seifert

Faculty of Engineering, Tel-Aviv University, ISRAEL
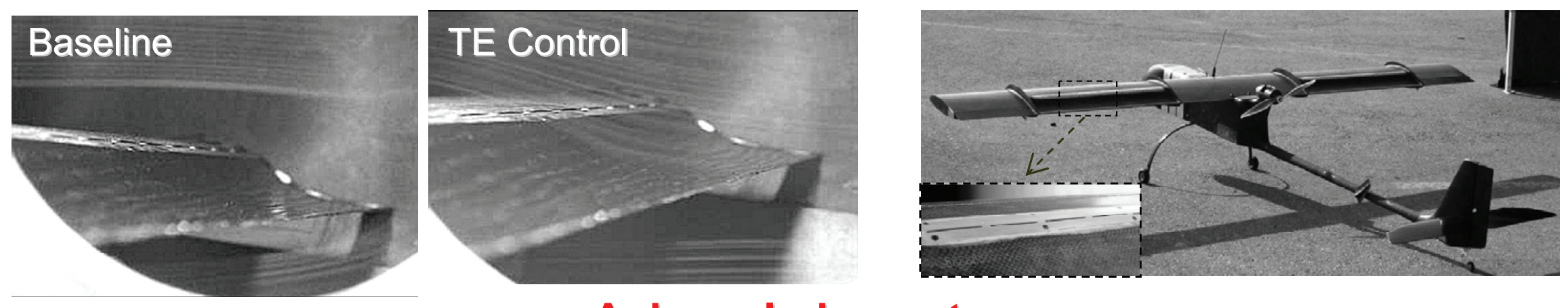

Acknowledgment:

I. Fono, G. Arwatz, T. Yehoshua, E. Ben-Hamou, I. Dayan S. Pasteur, E. Nevo, Kronish, Blas, Meadow Aero Lab members 


\section{Motivation}

- Active control of boundary layer separation leads to many benefits

- Frequency parameter: Strouhal $\left(F^{+}\right)$

- Length scale under controversy

- Amplitude scaling?

- We use $C_{\mu}$ - historically, steady blowing

- But does it scale the data?

- Are there other parameters we should consider?

- We revisit here known and define 2 new amplitude scaling parameters

- Check their validity using low Re data $\left(\operatorname{Re}<1 \times 10^{6}\right)$
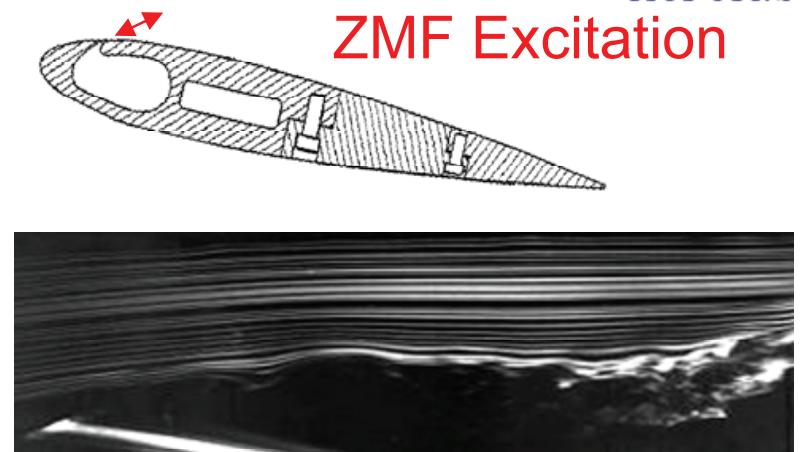

Baseline

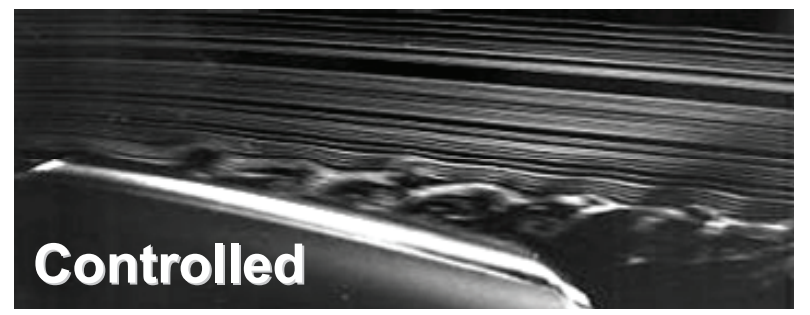

(Neuburger, '89, Re<100k)

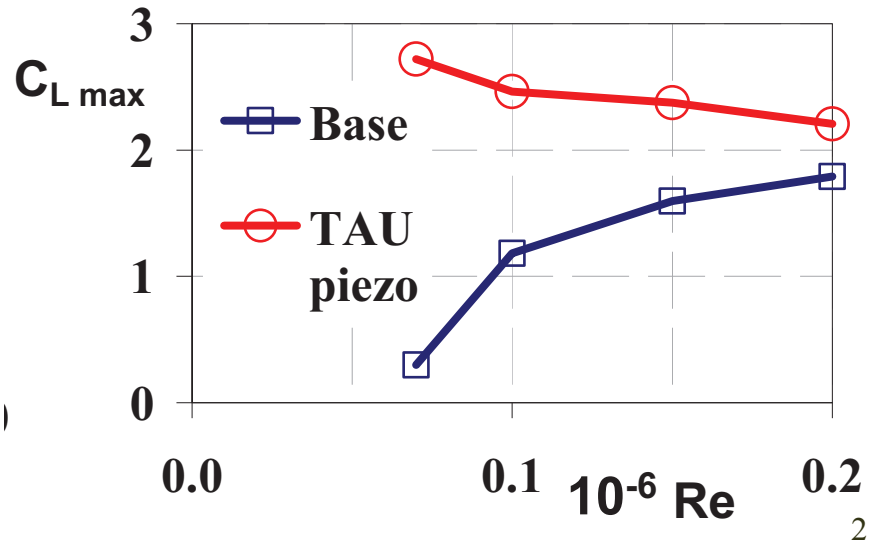




\section{Amplitude scaling - 1}

- Velocity Ratio

$$
V R \equiv \frac{U_{p}}{U_{e}} \approx \frac{U_{p}}{U_{\infty}}
$$

- $U_{p}$-peak excitation velocity

- $U_{e}$-local external velocity

- $U_{\infty}$ - free-stream velocity

- Actuator Mach number over freestream Mach number

- Implies linearity

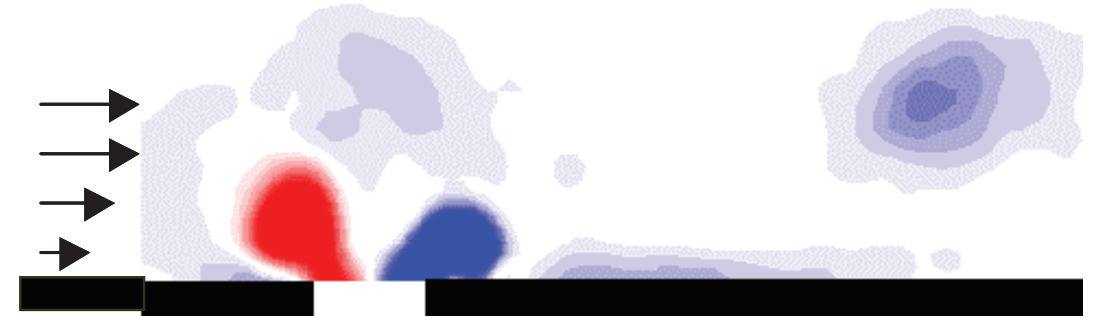

(Yehoshua) 


\section{Amplitude scaling - 2}

- Momentum coefficient

$$
C_{\mu} \equiv \frac{h}{c}\left(\frac{U_{p}}{U_{\infty}}\right)^{2}=\frac{h}{c}(V R)^{2}
$$

- Ratio between (incomp., isoth.)

- Excitation momentum ( $h=$ slot width)

$h U_{p}^{2}$

- Free stream momentum ( $c=c h o r d)$

$c U_{\infty}^{2}$

- $U_{p}$ - peak excitation velocity

- $U_{\infty}$ - free-stream velocity

- $h$ - slot width, $c$ - airfoil chord

- Follows steady-blowing concept

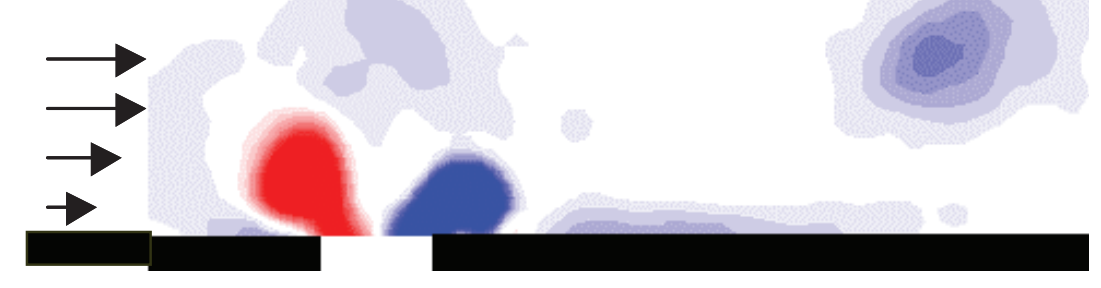




\section{Amplitude scaling - 3}

- Reynolds \# corrected momentum coefficient

- Ratio between

- Excitation momentum

$\approx h U_{p}^{2}$

- BL momentum deficit $\quad \approx \theta U_{\infty}^{2}$

- Laminar momentum thickness $\frac{\theta_{\text {Lam }}}{c} \approx \mathrm{Re}^{-0.5}$

- Turbulent momentum thickness

$\frac{\theta_{\text {Turb }}}{c} \approx \mathrm{Re}^{-0.2} \Rightarrow \theta_{\text {Turb }} \approx c \mathrm{Re}^{-0.2}$

$C_{\mu, \operatorname{Re}} \equiv \frac{h}{\theta} \frac{U_{p}^{2}}{U_{\infty}^{2}} \approx \frac{h(V R)^{2}}{c \operatorname{Re}^{m}}=\frac{h}{c}(V R)^{2} \mathrm{Re}^{-m}$ 


\section{Vorticity-flux / Circulation - 4}

- Actuator's vorticity flux (blowing, half slot width)

$$
V F \approx \int_{0}^{T / 2} \int_{0}^{h / 2} \omega_{z}(y, t) U_{p}(y, t) d y d t
$$

$$
V F \approx \int_{0}^{h / 2} \int_{0}^{T / 2} U_{p}(y, t) \frac{d U p(y, t)}{d y} d t d y
$$$$
V F \approx \frac{U_{p}^{2}}{h} \frac{h T}{4} \approx \frac{U_{p}^{2} T}{4} \approx \frac{U_{p}^{2}}{4 f}
$$

- Kutta-Zhukovsky

- Circulation

- Vorticity-flux coefficient
$C_{L}=\frac{\rho_{\infty} U_{\infty} \Gamma}{1 / 2 \rho_{\infty} U_{\infty}^{2} c}$

$\Gamma=c C_{L} U_{\infty}$

$C_{\Gamma} \equiv \frac{\text { Slot_Vorticity_Flux }}{\text { Baseline_Circulation }} \approx \frac{U_{p}^{2} T}{4 \Gamma_{\text {Baseline }}}$

$$
C_{\Gamma} \approx \frac{U_{p}^{2} T}{4 c C_{L} U_{\infty}}=\frac{1}{4 C_{L}}\left(\frac{U_{p}}{U_{\infty}}\right)^{2} \frac{1}{S_{t}}=\frac{(V R)^{2}}{4 C_{L} S_{t}}
$$




\section{Frequency corrected VR - 5}

- Nagib et al (2006)

- Proposed to scale AFC effect on lift using

$$
H \equiv \frac{U_{p} / U_{\infty}}{\sqrt{f_{c} / U_{\infty}}}=\frac{V R}{\sqrt{S_{t}}}
$$

- Velocity ratio

- Strouhal number

$\stackrel{\omega}{\underline{\Xi}}$

- For large separated regions

- Low frequencies

- Problematic at $f \rightarrow 0 \ldots$

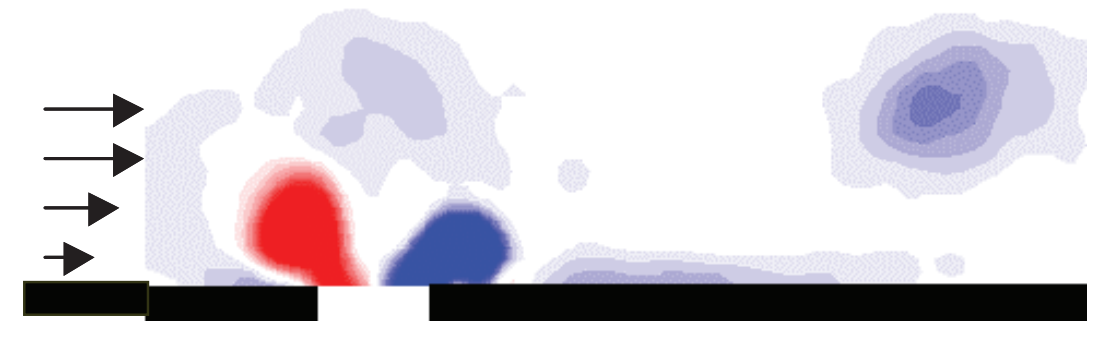

(Yehoshua) 


\section{Amplitude Scaling Options}

- Velocity ratio (VR)

$$
V R \equiv \frac{U_{p}}{U_{e}} \approx \frac{U_{p}}{U_{\infty}}
$$

- Strouhal weighted VR (Nagib et al, 2006)

$$
H \equiv \frac{V R}{\sqrt{S_{t}}}
$$

$$
C_{\mu} \equiv \frac{h}{c}(V R)^{2}
$$

- Reynolds corrected $C_{\mu}$

- $m=0.2$ (Turb.), $m=0.5$ (Lam.)

$$
\begin{aligned}
C_{\mu, \operatorname{Re}} & \equiv \frac{h}{c}(V R)^{2} \\
C_{\Gamma} & \equiv \frac{(V R)^{2}}{C_{L} S_{t}}
\end{aligned}
$$

- Vorticity flux coefficient 


\section{IAI-Pr8-40 Airfoil AFC Testing}

梌

- Chord $360 \mathrm{~mm}$, span $609 \mathrm{~mm}$

- Actuator at $\mathrm{x} / \mathrm{c}=\mathbf{0 . 3 8}$

- Shallow angle, downstream excitation

- 14 segmented, individually controlled

- Piezo actuators $\left(f_{\text {Helmholtz }}=1460 \mathrm{~Hz}\right)$

- Slots: $0.9 \mathrm{~mm}$ by $40 \mathrm{~mm}$

- Power: $1.7 \mathrm{w}$ per actuator at $100 \mathrm{vac}$
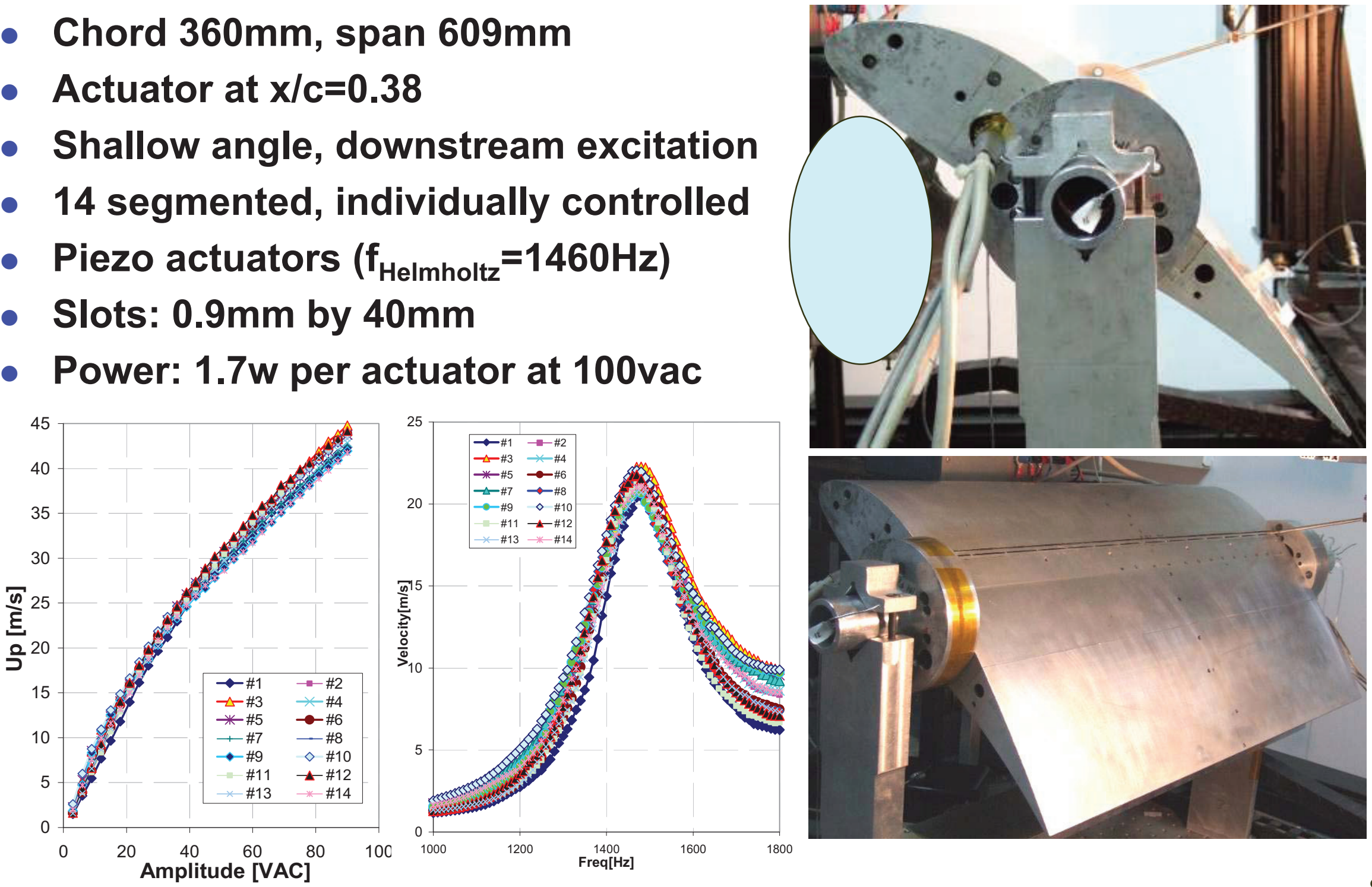


\section{|A|-Pr8-40 Baseline}

- Thick, Efficient, Low Re airfoil

- Mild, trailing edge stall

- Chord $360 \mathrm{~mm}$, span $609 \mathrm{~mm}$

- Actuator at $\mathbf{x} / \mathbf{c}=0.38$

- Shallow angle, downstream excitation

- 14 segmented, individually controlled

- Piezo actuators $\left(f_{\text {Helmholtz }}=1460 \mathrm{~Hz}\right)$

- Slots: $0.9 \mathrm{~mm}$ by $40 \mathrm{~mm}$

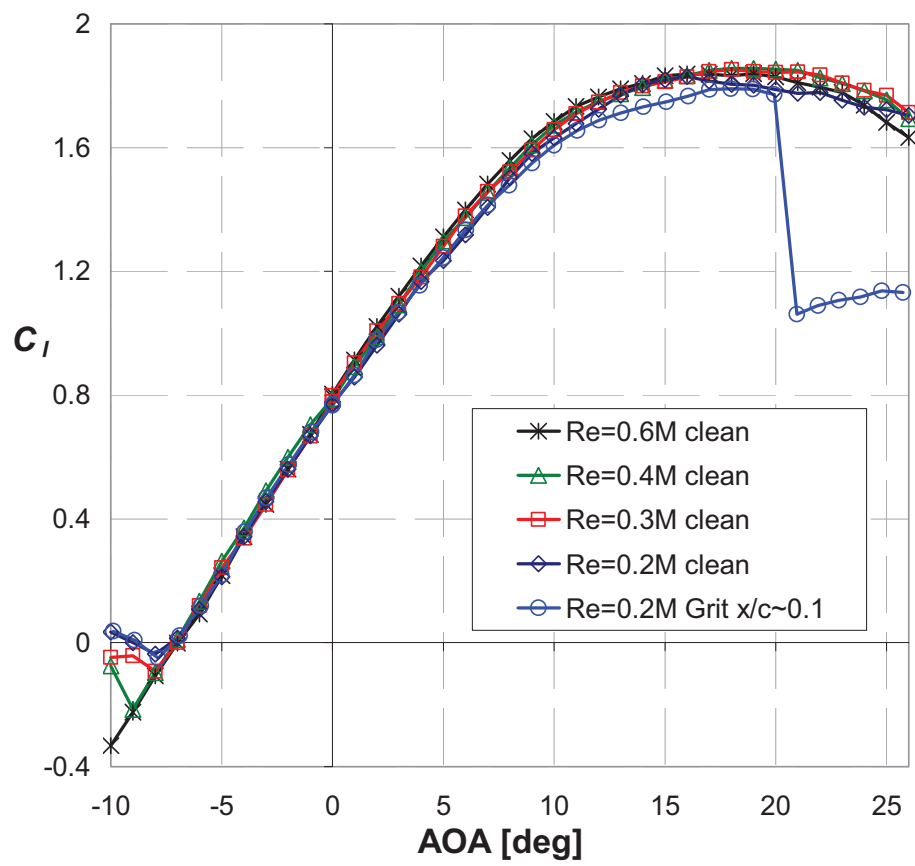

$\mathrm{C}_{\mathrm{P}}$
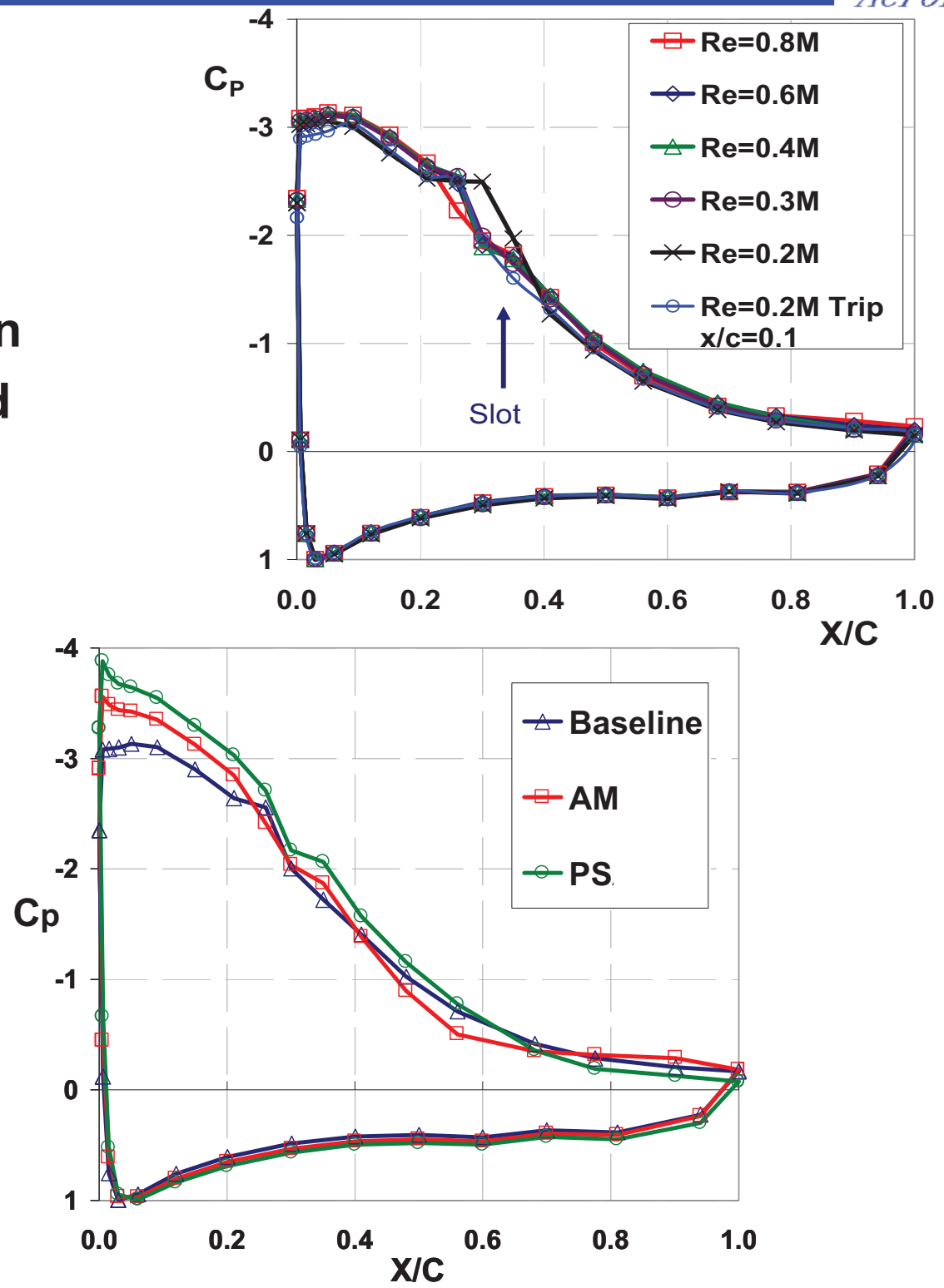


\section{IAI-Pr8-40 Controlled}

- Actuator at $\mathrm{x} / \mathrm{c}=\mathbf{0 . 3 8}$

- Shallow angle, downstream excitation

- 14 segmented, individually controlled

- Piezo actuators $\left(f_{\text {Helmholtz }}=1460 \mathrm{~Hz}\right)$

- Slots: $0.9 \mathrm{~mm}$ by $40 \mathrm{~mm}$

- Two modes of operation:

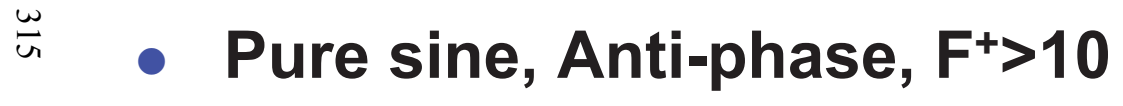

- AM, In-phase, $F^{+} \sim 1$

- Control of TBL separation

- Conducted amplitude scans at otherwise fixed conditions

- Scale lift increment vs amplitude

AM
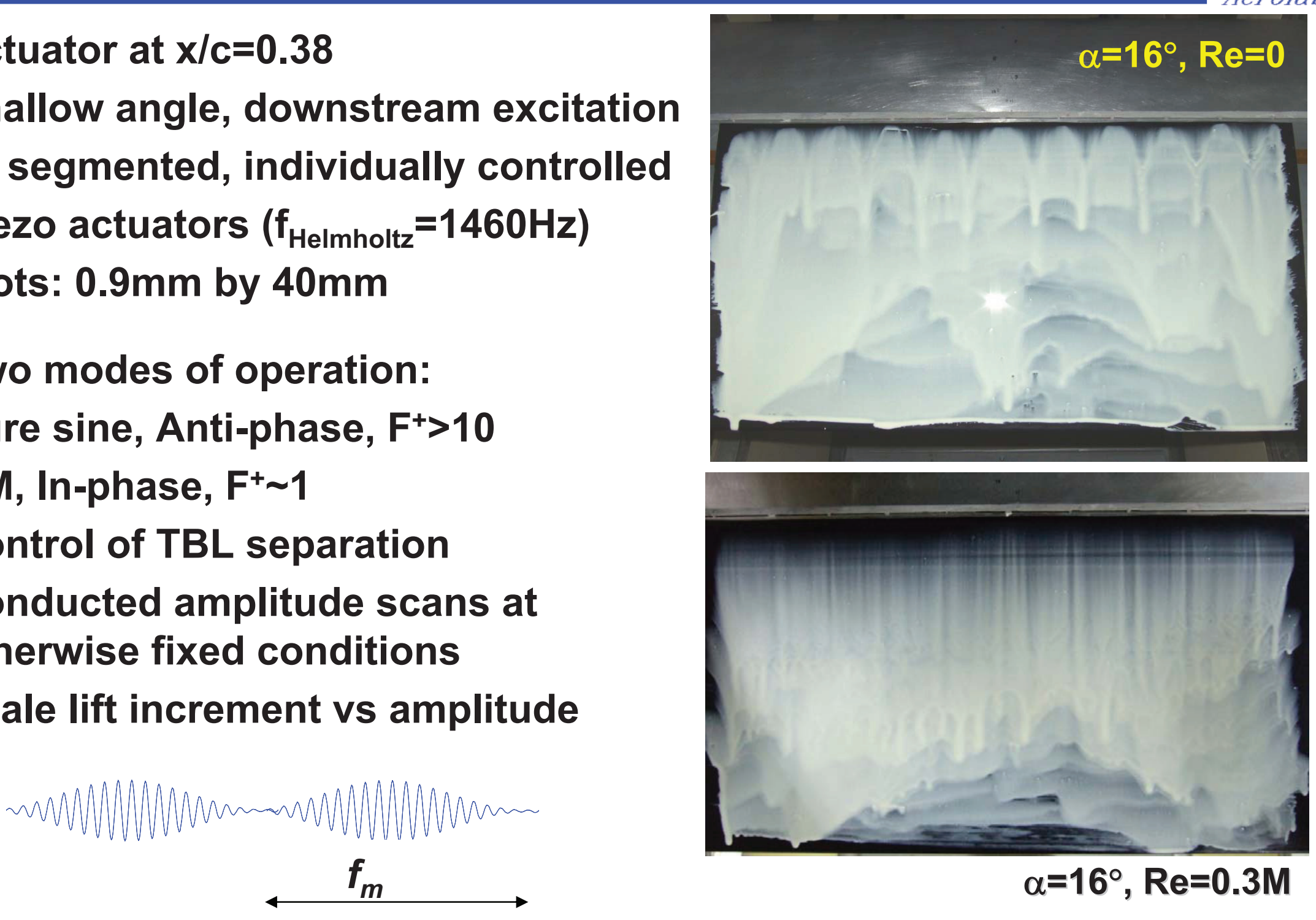

$\alpha=16^{\circ}, \operatorname{Re}=0.3 \mathrm{M}$ 


\section{to Low Frequency Amp Scaling}

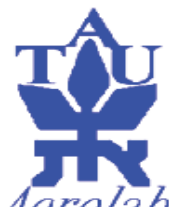

- Velocity ratio (VR)

$\alpha=16^{\circ}, A M, F+=1$

$\ddot{\omega}$

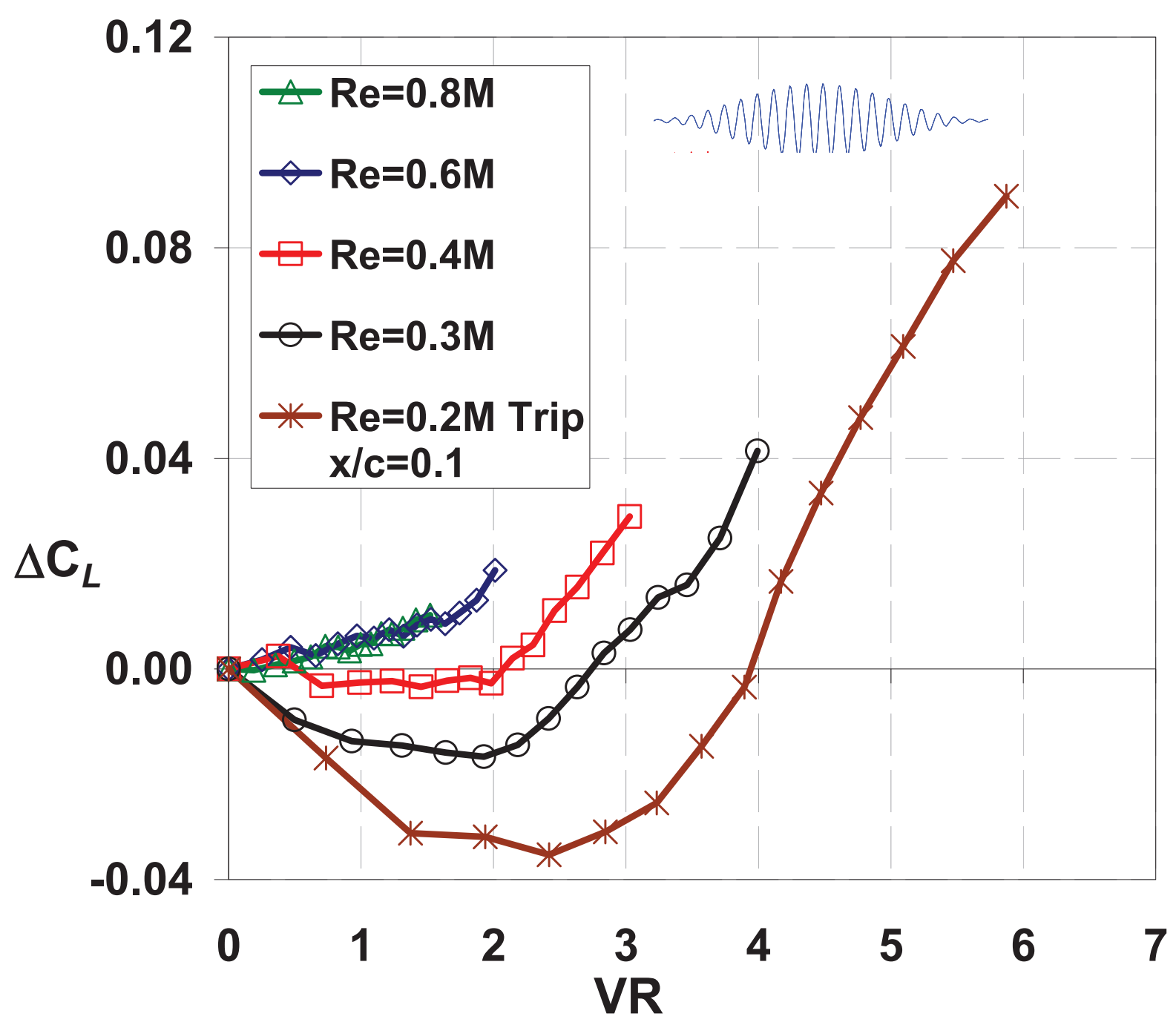




\section{Low Frequency Amp Scaling}

$\alpha=16^{\circ}, A M, F+=1$

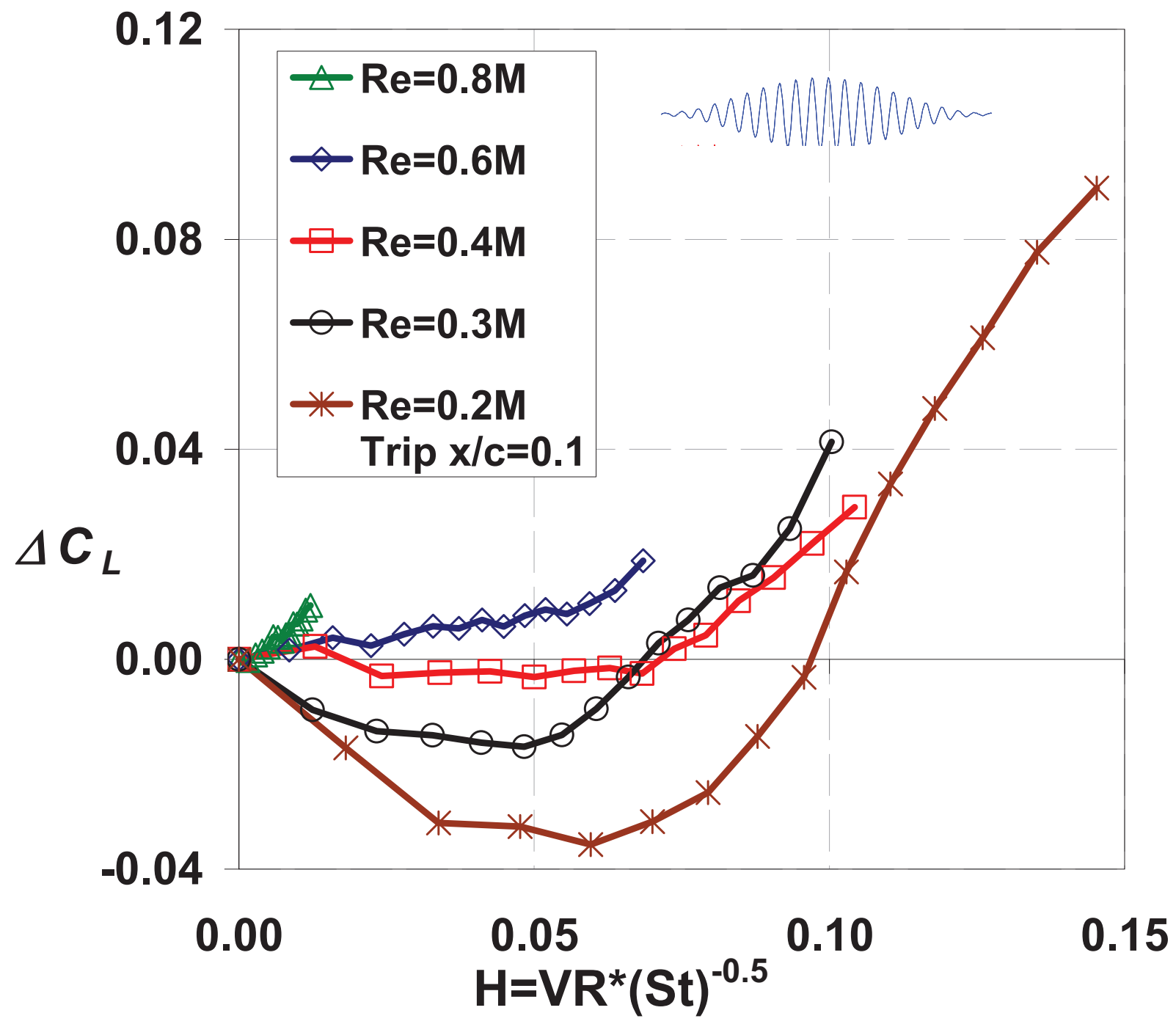




\section{Lew Frequency Amp Scaling}

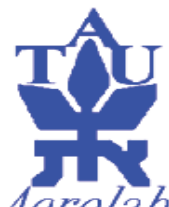

- Momentum Coefficient

$\alpha=16^{\circ}, A M, F+=1$

$\underset{\infty}{\infty}$

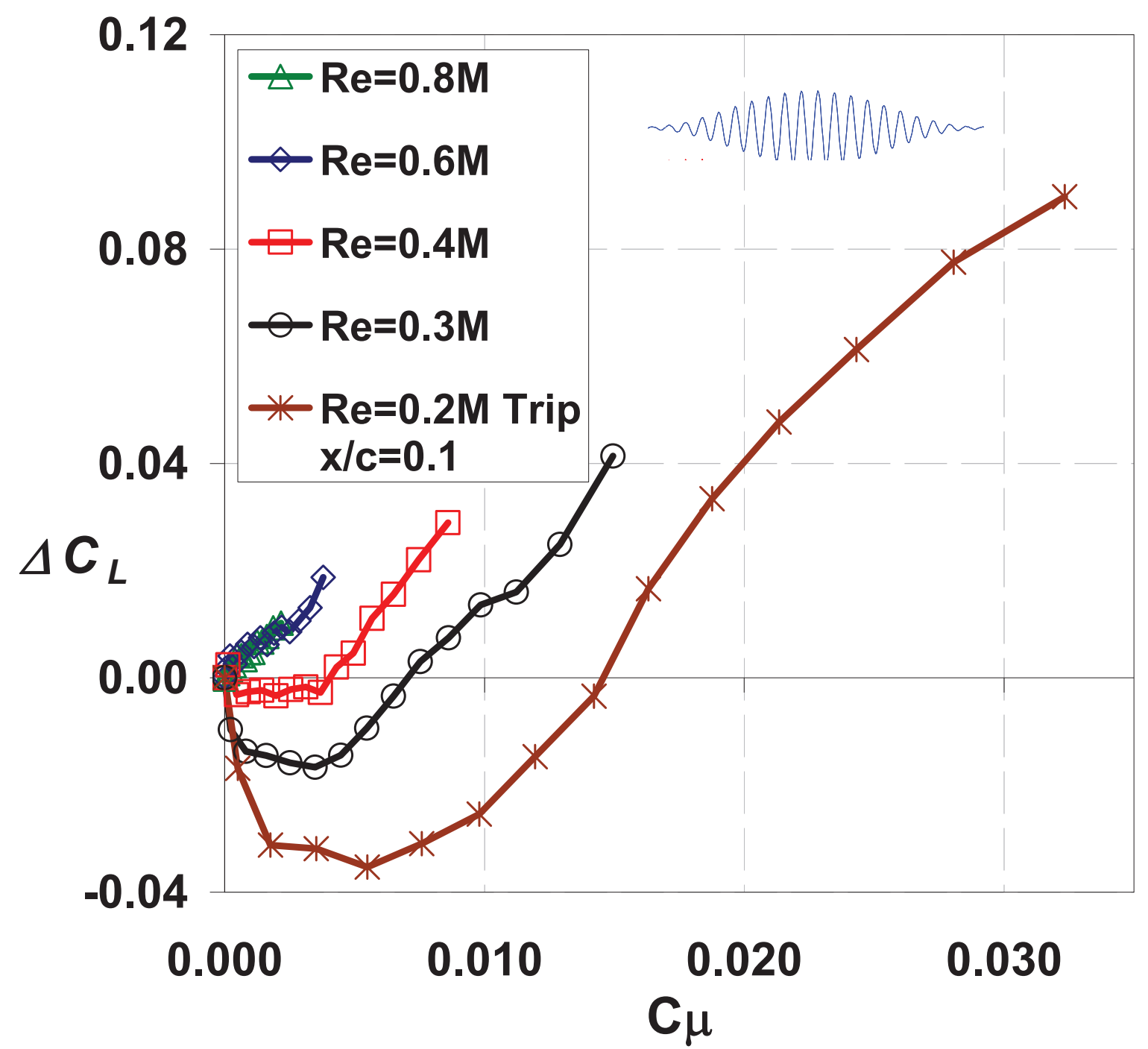




\section{Low Frequency Amp Scaling}

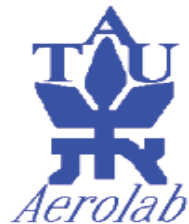

- Reynolds corrected Momentum Coefficient $\alpha=16^{\circ}, A M, F+=1$

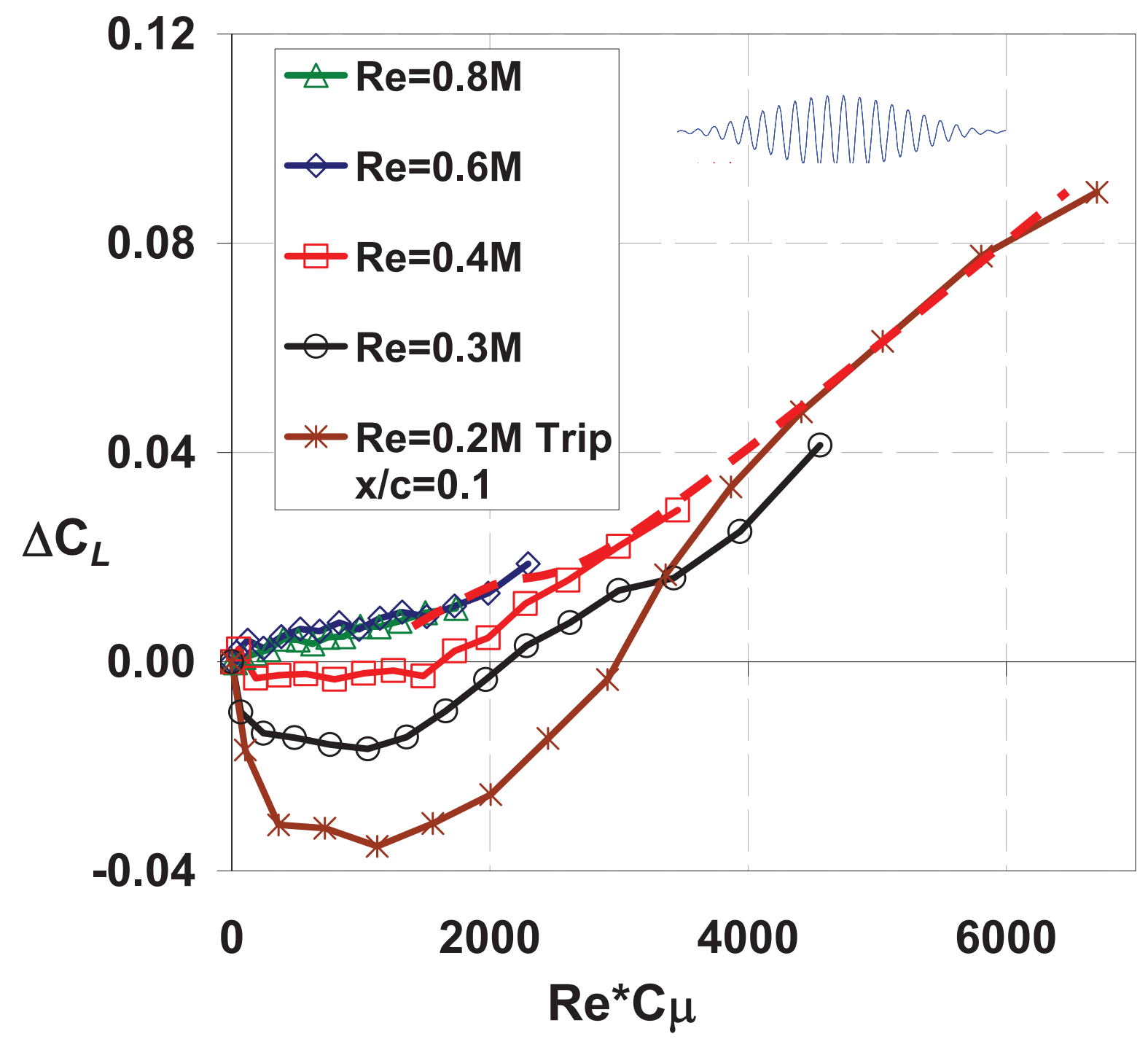




\section{. 250- Low Frequency Amp Scaling}

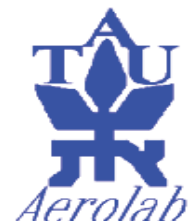

- Vort. 0.12 Flux Coeff.

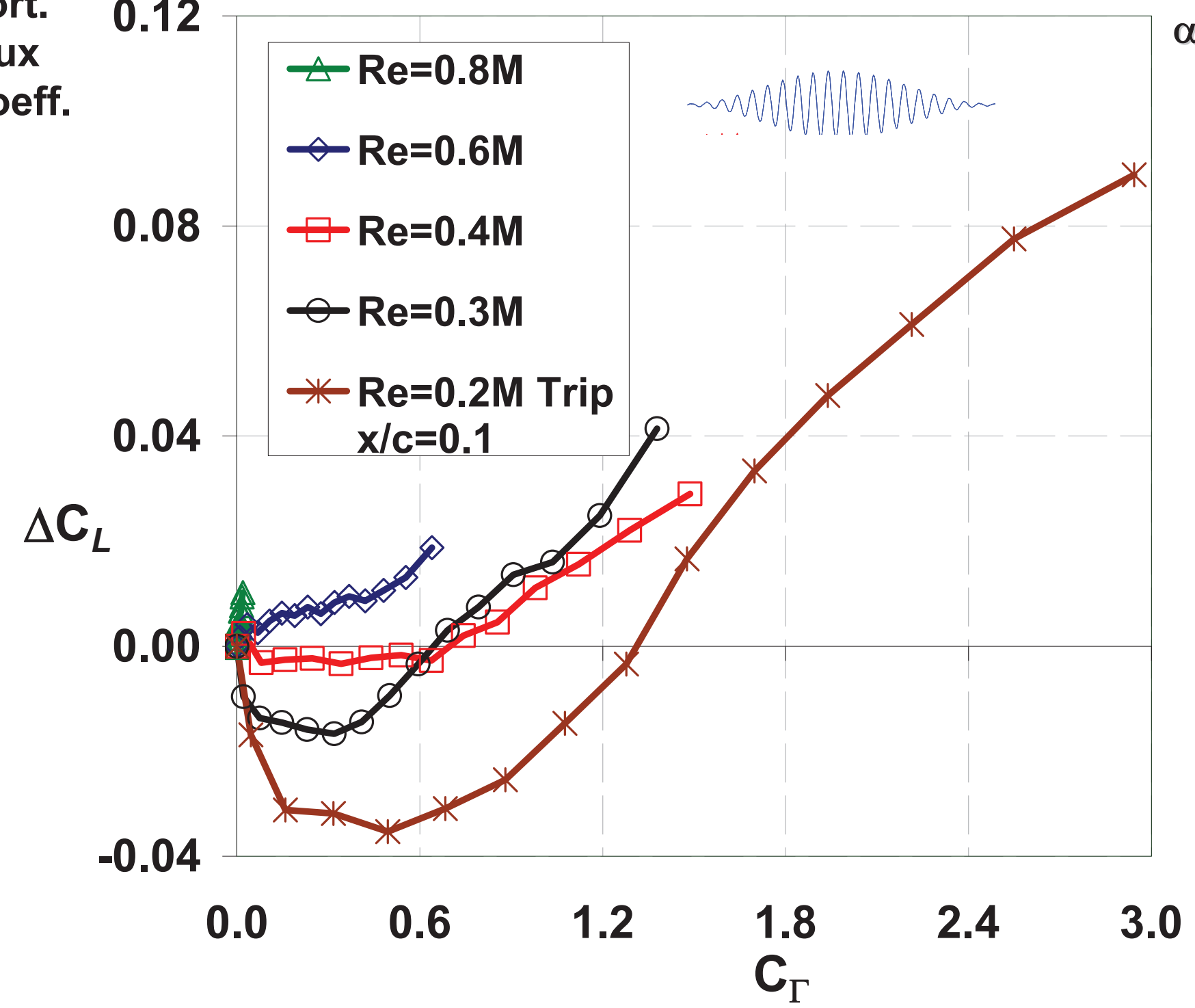




\section{High Frequency Amp Scaling}

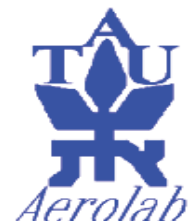

ํㅗㅇ

- Velocity ratio

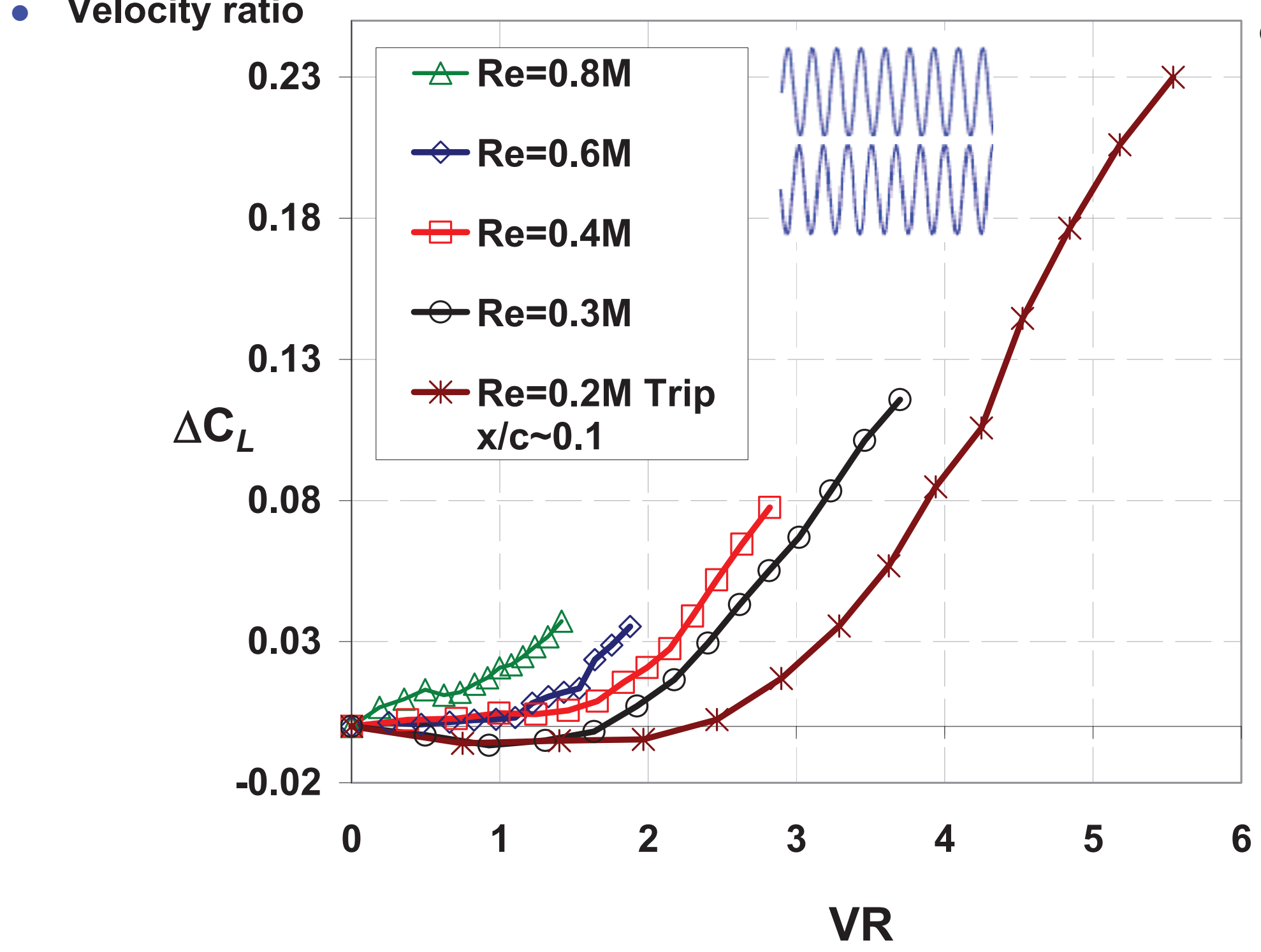
$\alpha=16^{\circ}, \mathrm{PS}, \mathrm{Pi}$

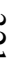




\section{High Frequency Amp Scaling}

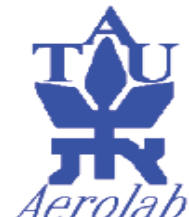

- St corrected VR

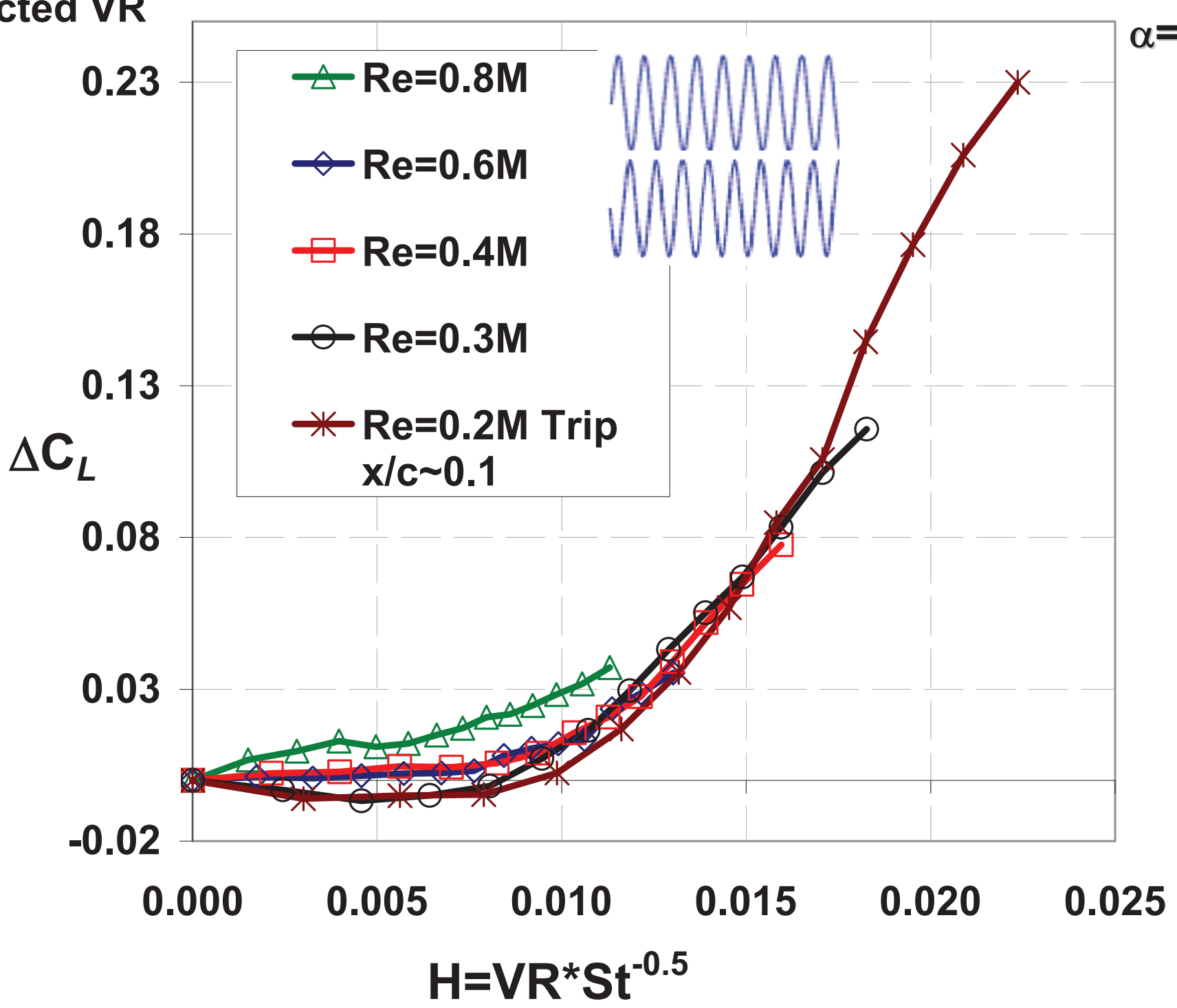

$\alpha=16^{\circ}, \mathrm{PS}, \mathrm{Pi}$

$$
\mathrm{H}=\mathrm{VR}^{*} \mathrm{St}^{-0.5}
$$




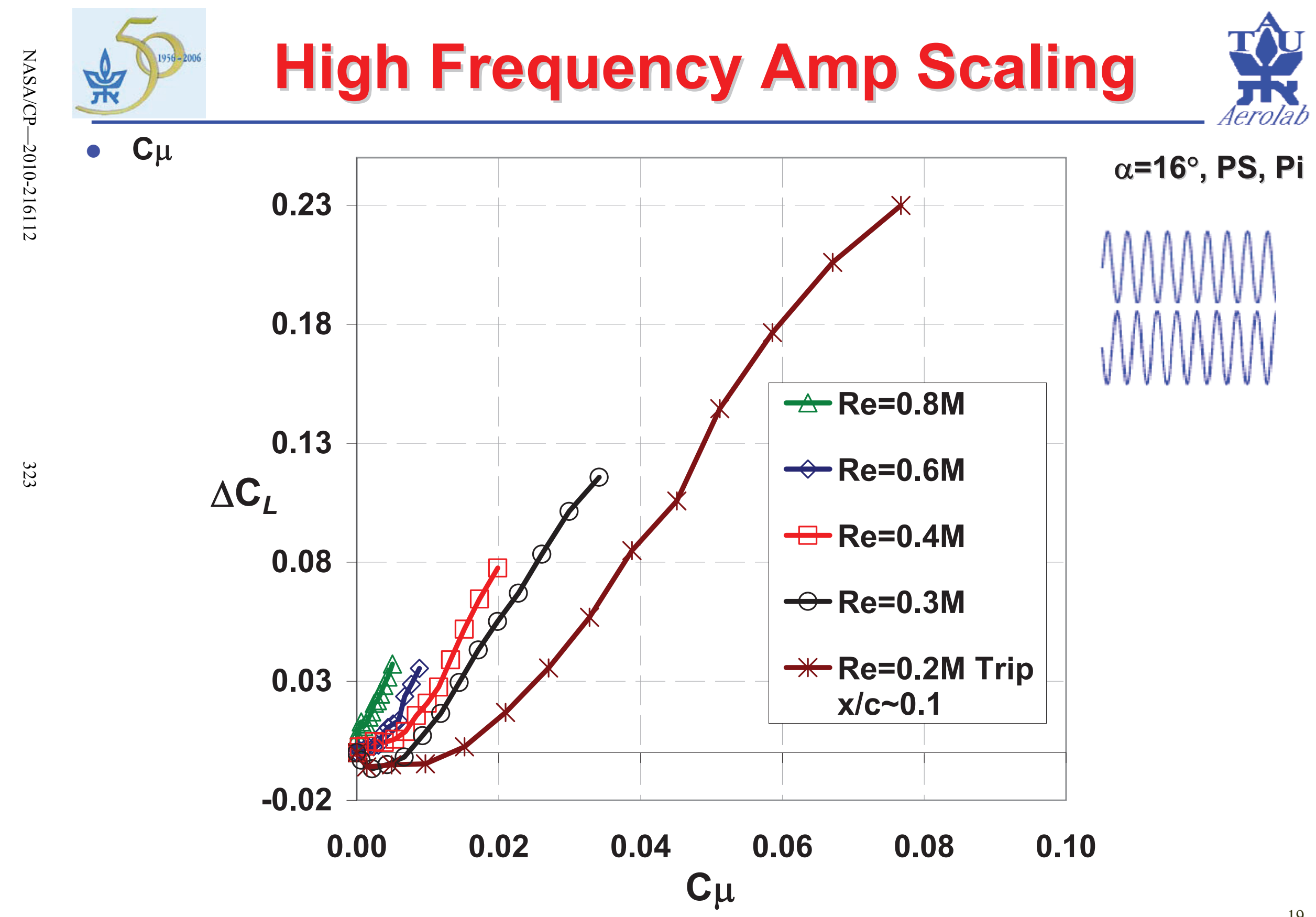




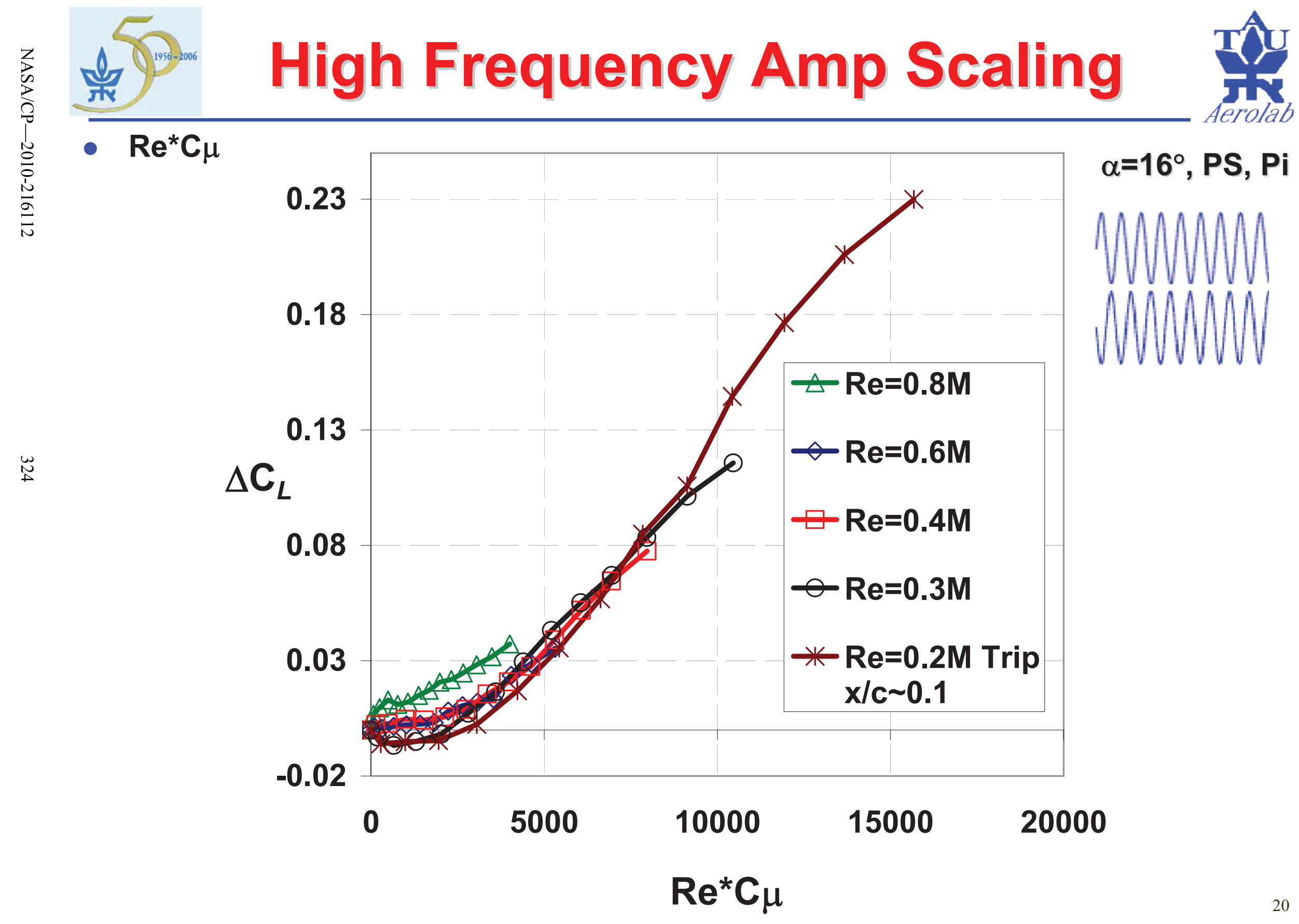




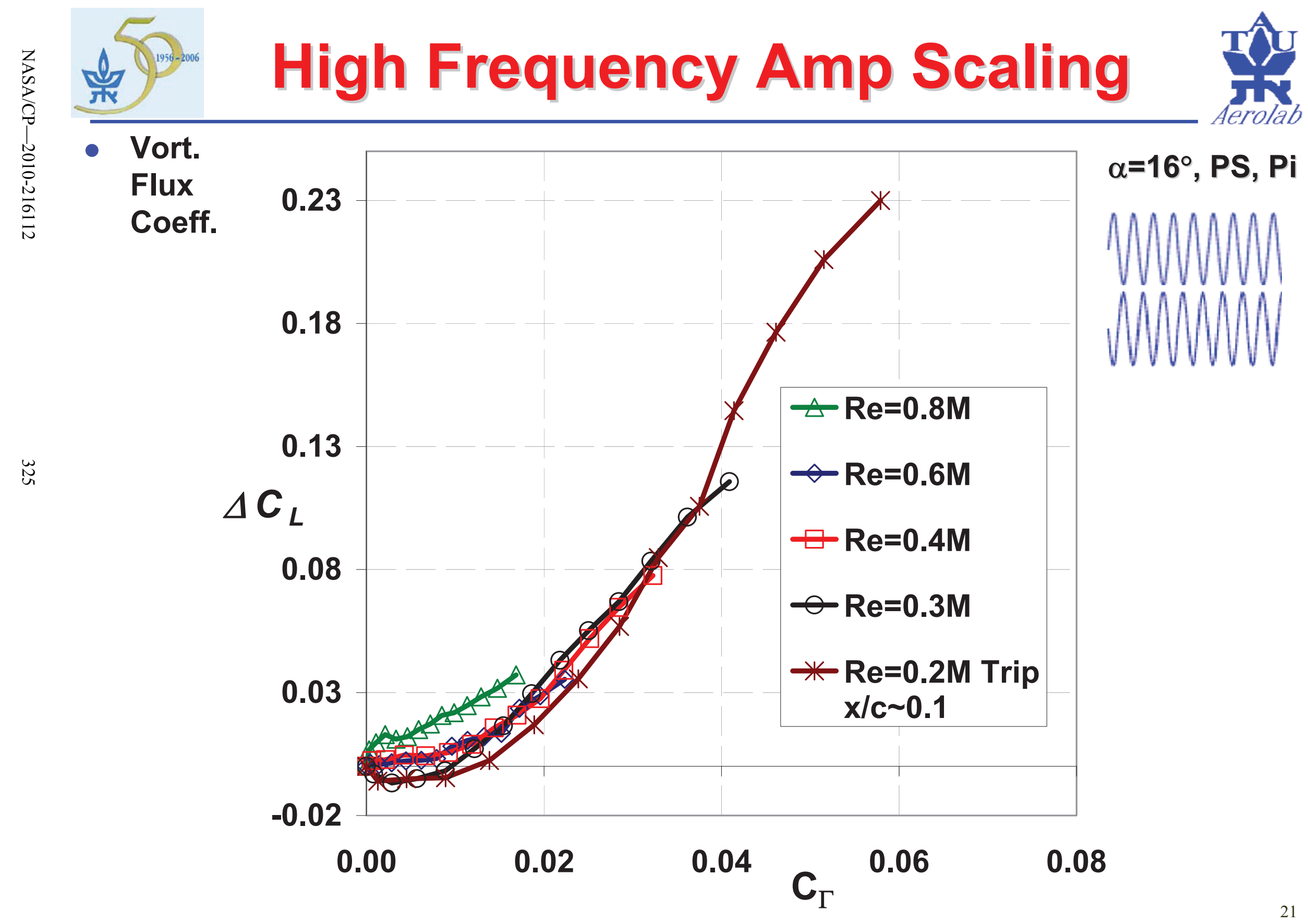




\section{Amplitude Scaling Summary}

- Velocity ratio (VR)

$$
V R \equiv \frac{U_{p}}{U_{e}} \approx \frac{U_{p}}{U_{\infty}}
$$

$\mathrm{F}^{+}=1$

$F^{+}>10$

- Strouhal weighted VR (Nagib et al, 2006)

$$
H \equiv \frac{V R}{\sqrt{S_{t}}}
$$

$\mathbf{X}$

- Momentum coefficient

$$
C_{\mu} \equiv \frac{h}{c}(V R)^{2}
$$

- Reynolds corrected $C_{\mu}$

- $m=0.2$ (Turb.), $m=0.5$ (Lam.)

$$
\begin{gathered}
C_{\mu, \mathrm{Re}} \equiv \frac{h}{c}(V R) \\
C_{\Gamma} \equiv \frac{(V R)^{2}}{C_{L} S_{t}}
\end{gathered}
$$

$\sim \sqrt{ }$

- used $m=1$...

- Vorticity flux coeff. 


\section{Conclusions}

- Three existing and two new excitation magnitude scaling options for active separation control at Reynolds numbers below one Million.

- The physical background for the scaling options was discussed and their relevance was evaluated using two different sets of experimental data.

- For $F^{+} \sim 1,2 D$ excitation:

- The traditional $V R$ and $C \mu$ - do not scale the data

- Only the $\operatorname{Re}^{*} C \mu$ is valid

- This conclusion is also limited for positive lift increment.

- For $F^{+}>10,3 D$ excitation, the Re corrected $C \mu$, the $S t$ corrected velocity ratio and the vorticity flux coefficient, all scale the amplitudes equally well.

- Therefore, the Reynolds weighted $C \mu$ is the preferred choice, relevant to both excitation modes.

- Incidence also considered, using Ue from local Cp 


\section{Vorticity flux ratio - 4}

- Actuators also generate vorticity flux and alter circulation

$$
C_{V F} \equiv \frac{\left[U_{p}(t, y) \frac{d U_{p}(t, y)}{d y}\right]_{\max }}{\left[U_{B L}(y) \frac{d U_{B L}(y)}{d y}\right]_{\max }} \approx \frac{U_{p}^{2}}{h / 2} \frac{\theta}{U_{e}^{2}}=\frac{2 \theta}{h}\left(\frac{U_{p}}{U_{e}}\right)^{2} \approx \frac{0.01 c}{h}\left(\frac{U_{p}}{U_{e}}\right)^{2}
$$

$\underset{\infty}{\sim}$

- While the momentum coefficient

- For $h / c=0.005$ (and $\theta / c=0.005$ )

$$
C_{\mu} \equiv \frac{2 h}{c}\left(\frac{U_{p}}{U_{e}}\right)^{2}
$$

- So the ratio $C_{V F} / C_{\mu} \equiv \frac{0.01 c}{h} / \frac{2 h}{c}=\frac{0.005 c^{2}}{h^{2}} \approx \frac{5 \times 10^{-3}}{2.5 \times 10^{-5}}=200$

- Is very large; define Vorticity Flux Coefficient 


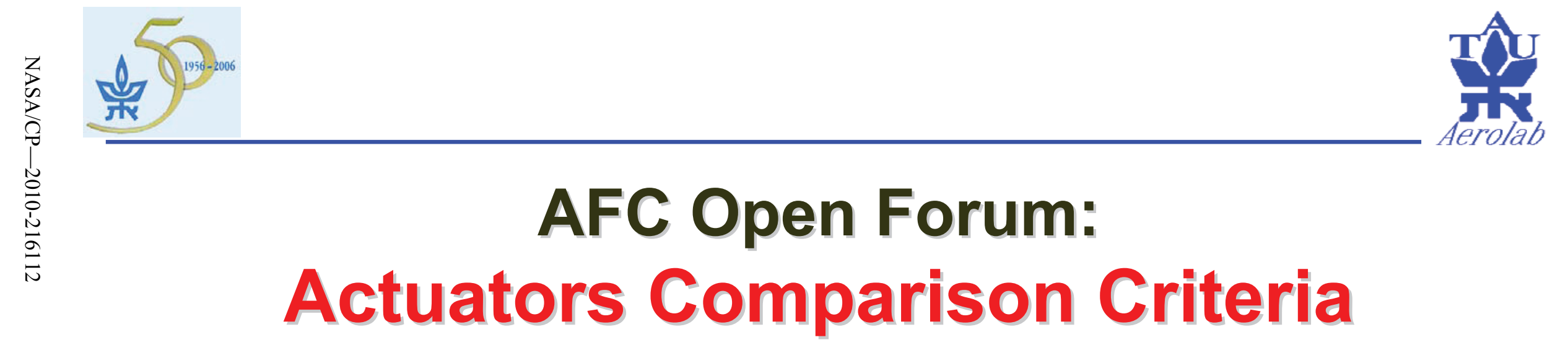

\section{Avraham "Avi" Seifert}

Acknowledgment:

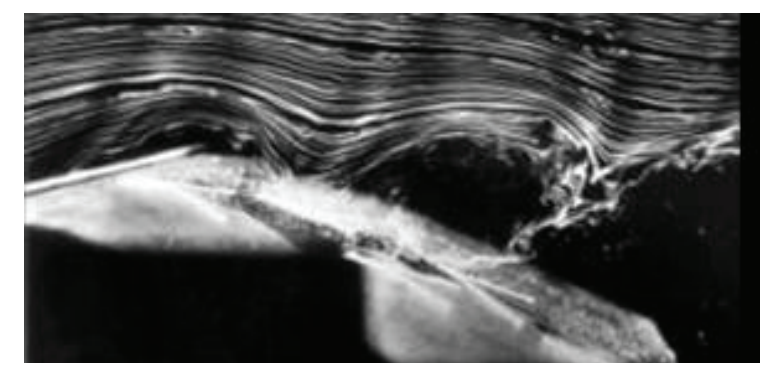

I. Fono, G. Arwatz, T. Yehoshua, O. Stalnov, E. Ben-Hamou I. Dayan, S. Paster, E. Nevo, Meadow Aero Lab members 


\section{Motivation}

- Active control of boundary layer separation

- Actuation: key enabling technology

- Many actuator types exist

- No accepted criteria for actuator comparison

- Rare to find:

- Energy, efficiency, weight, cost data
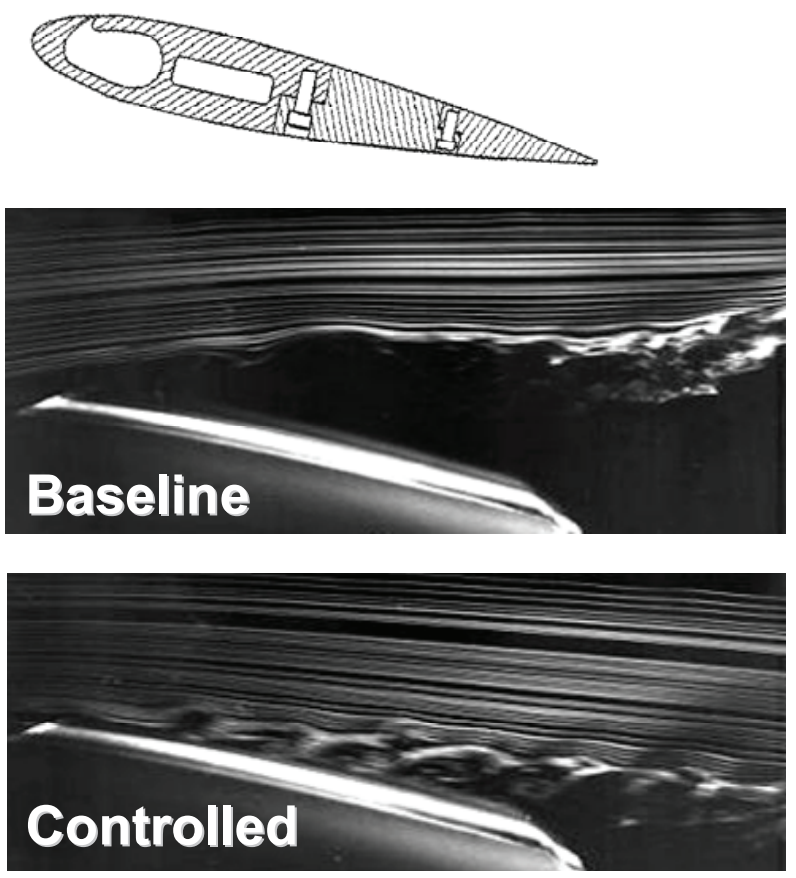

(Neuburger, '89, $\operatorname{Re}<100 k$ ) 


\section{Comparison criteria: OFM}

- Overall Figure of Merit

- Actuator operates in still air

$$
O F M \equiv \frac{F_{a}^{2} U_{p}}{W_{a} P}
$$

- $F_{a}$-force generated

- When not/can not be measured could be estimated by

$$
F_{a} \approx C \rho A_{a} U_{p}^{2}
$$

- With $C$ from 0.25 to 0.5 (blowing only, velocity profile)

- $U_{p}$-peak generated velocity

- $W_{a}$ - actuator weight

- $P$ - actuator energy consumption

- Electric, fluidic, combined

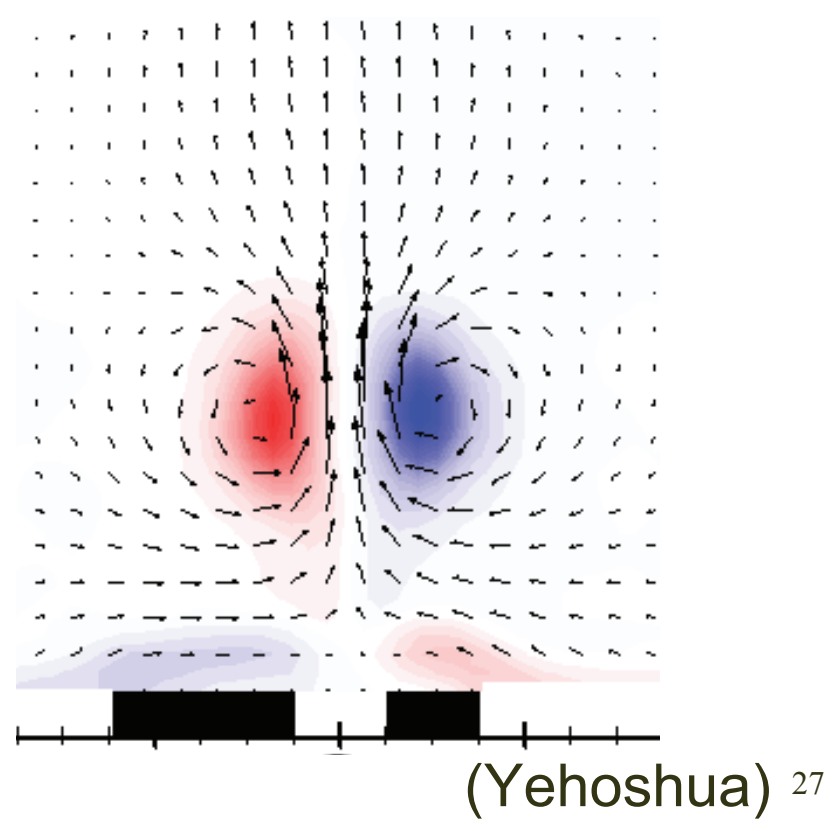




\section{Comparison criteria: AFM-1}

- Aerodynamic Figure of Merit (1)

$A F M 1 \equiv \frac{c /\left(U_{\infty} D_{c}+P\right)}{(L / D)_{\text {baseline }}}$

- Application, actuators and flow condition dependent

- $U_{\infty}$ - Free-stream velocity

- $L$ - lift (baseline or controlled)

- $D$ - Drag (baseline or controlled)

- $P$ - actuator energy consumption

- Situation: Have system and actuators

- Question: Direct energy into power-plant or actuators?

- Answer: Only when $\boldsymbol{A F M 1}>1$ operate actuators 


\section{AFM-1 Example: 3D PZE}

- Low Re Control of separation Flow

- TAU Developed piezo-benders

- Considered energy efficiency

- Found that 3D excitation more effective than 2D

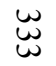
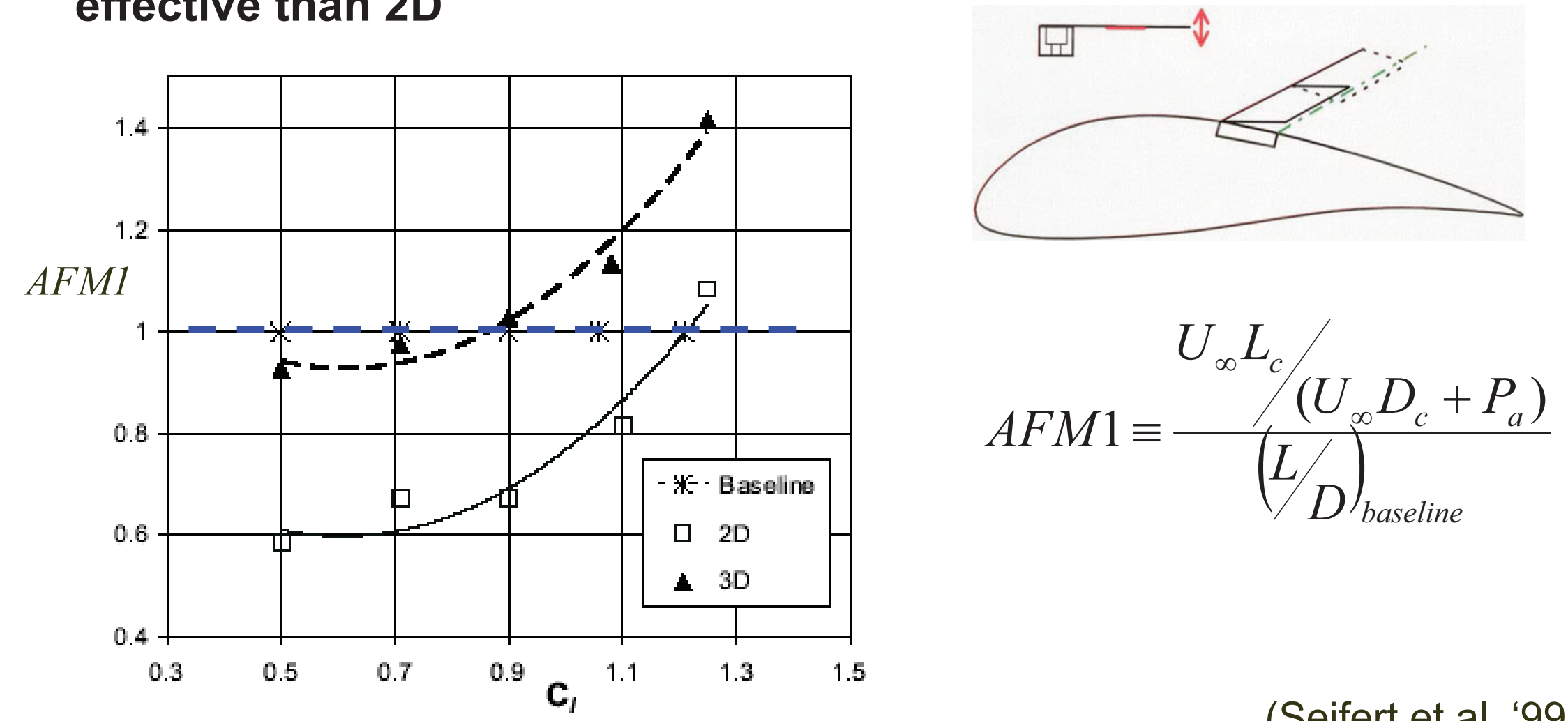

(Seifert et al, '99) 


\section{Comparison criteria: AFM-2}

- Aerodynamic Figure of Merit (2)

$$
\text { AFM2 } \equiv \frac{U_{\infty}\left(L_{c}-W_{a}\right) /\left(U_{\infty} D_{c}+P\right)}{(L / D)_{\text {baseline }}}
$$

- Application, actuators and flow condition dependent

- $U_{\infty}$ - Free-stream velocity

出 $L$ - lift (baseline or controlled)

- $D$ - Drag (baseline or controlled)

- $W_{a}$ - weight of actuation system (incl. drivers, cables...)

- $P$ - actuation SYSTEM energy consumption

- Situation: Scaled $A F M 1>1$ and actuation system weight known (or predicted)

- Question: Should we include actuation in system?

- Answer: only when AFM2 >1 (probably 1.1 minimum) 


\section{Actuation Methods}

\section{(Not a tutorial nor a comprehensive review)}

- External

- Speakers on tunnel walls

- Speakers in cavities

㟧 - Mechanical rotary

- Mechanical - pneumatic

- Ribbons + shakers

- Internal... 


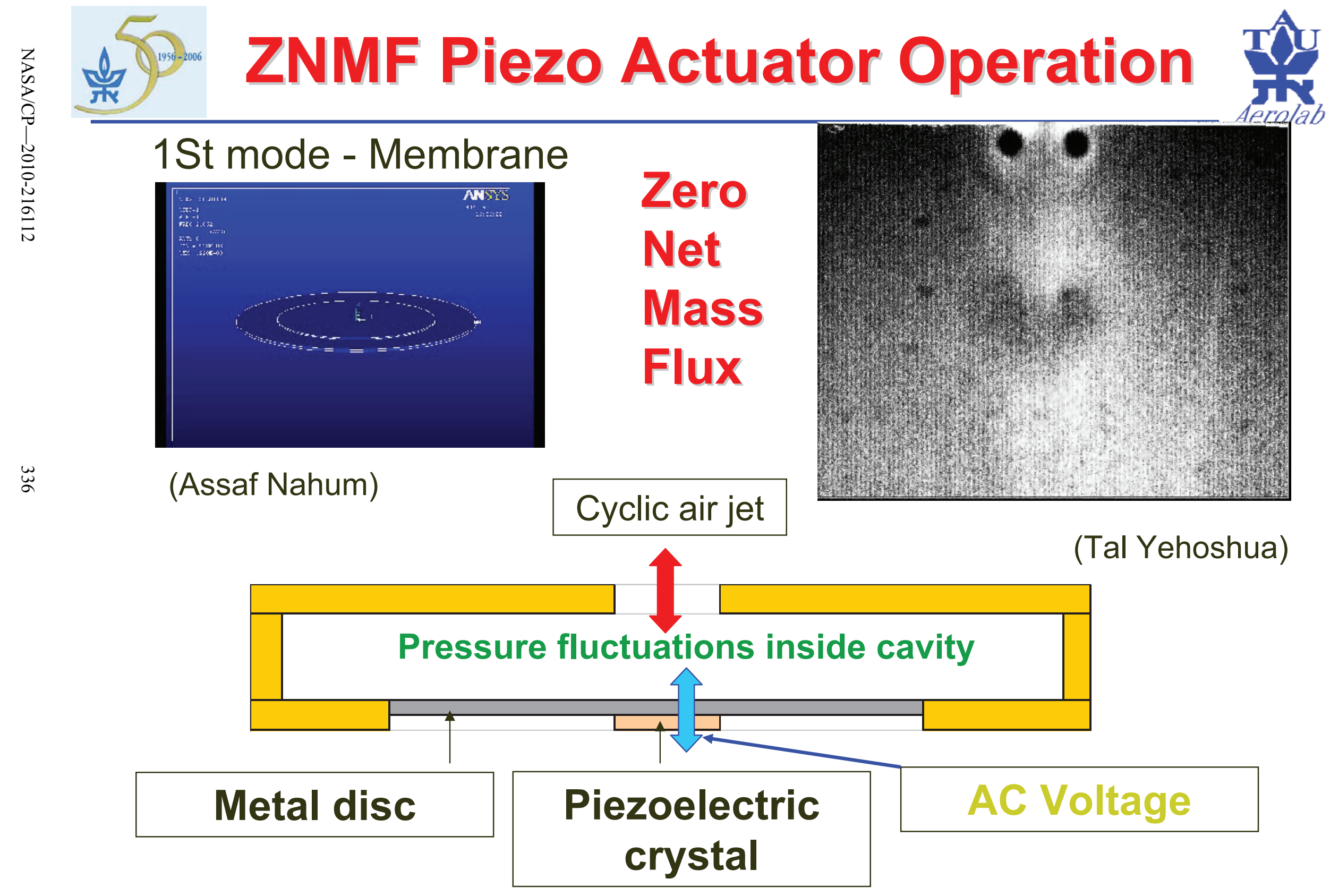




\section{Surface Mechanical Actuators}

- Requirement: Sufficient control authority (....any type)

- Type (affecting method and feasibility of characterization)

- Mechanical: Amplitude, Mode shape...

- Micro balloons, micro flaps...
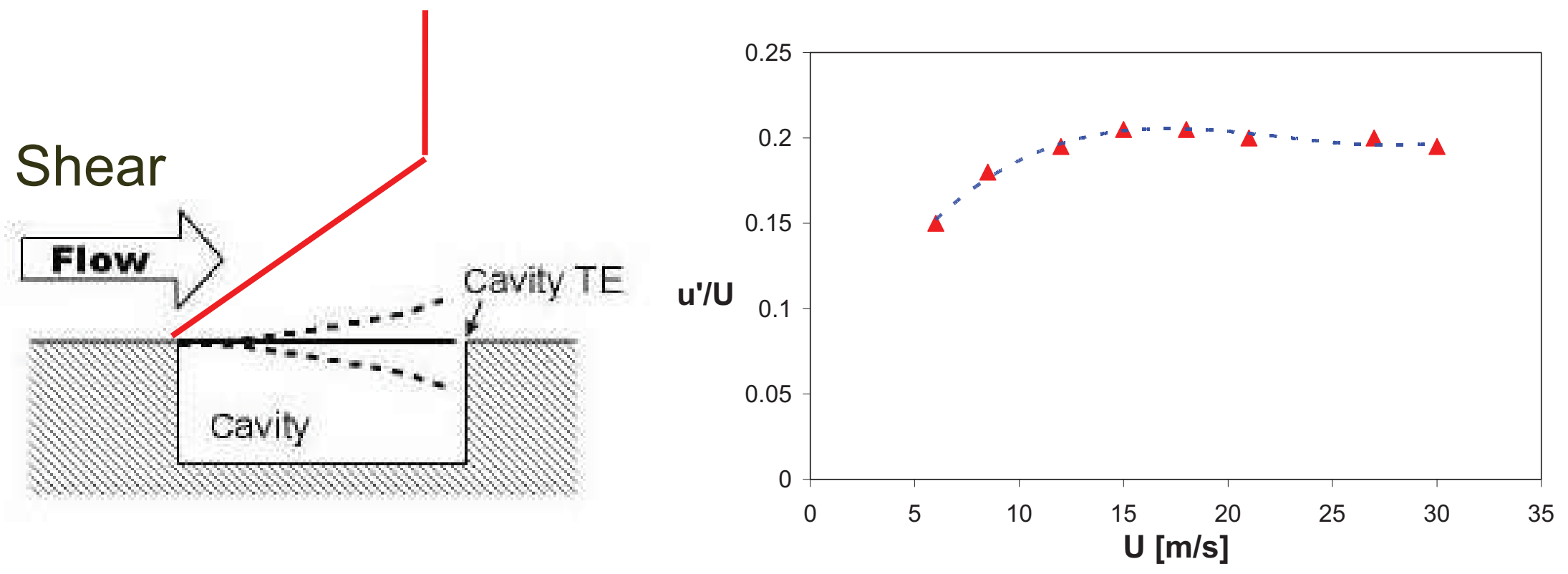

(Seifert et al., 1998, AlAA J., V. 36, N. 8.) 


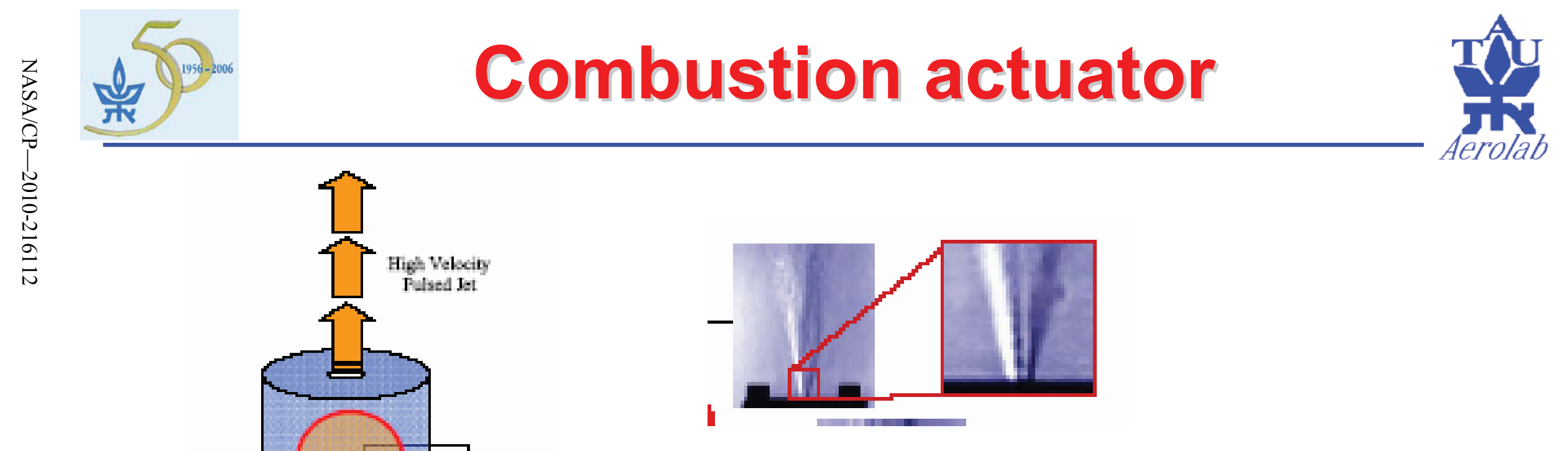

T. Crittenden and A. Glezer Woodruff School of Mechanical Engineering 801 Ferst Drive N.W.

E. Birdsell and M. Allen School of Electrical and Computer Engineering 777 Atlantic Drive N.W. Georgia Institute of Technology, Atlanta, GA, USA 30332 


\section{Surface Plasma actuator}

$U=0.0 \mathrm{kV} p-\mathrm{p}, P=0.0 \mathrm{~W} / \mathrm{m}, \mathrm{f}=0.0 \mathrm{kHz}$
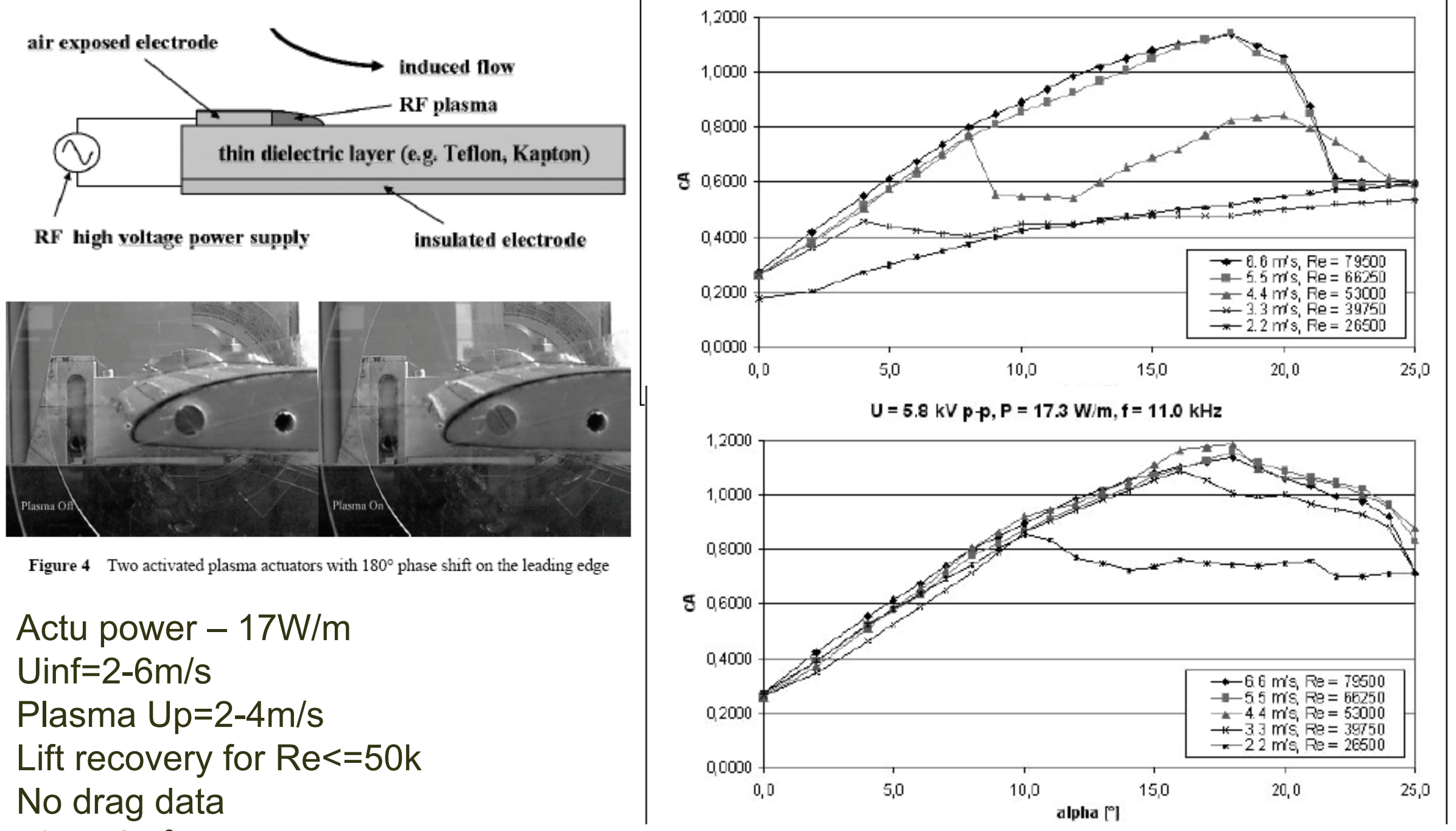

Actu power - 17W/m

Uinf $=2-6 \mathrm{~m} / \mathrm{s}$

Plasma Up=2-4m/s

Lift recovery for $\mathrm{Re}<=50 \mathrm{k}$

No drag data

$1 / 2 \operatorname{RoSU}^{3}=4.4 \mathrm{Watt}$

Active Flow Control by Surface Smooth Plasma Actuators

B. GÖKSEL, I. RECHENBERG, TUB 


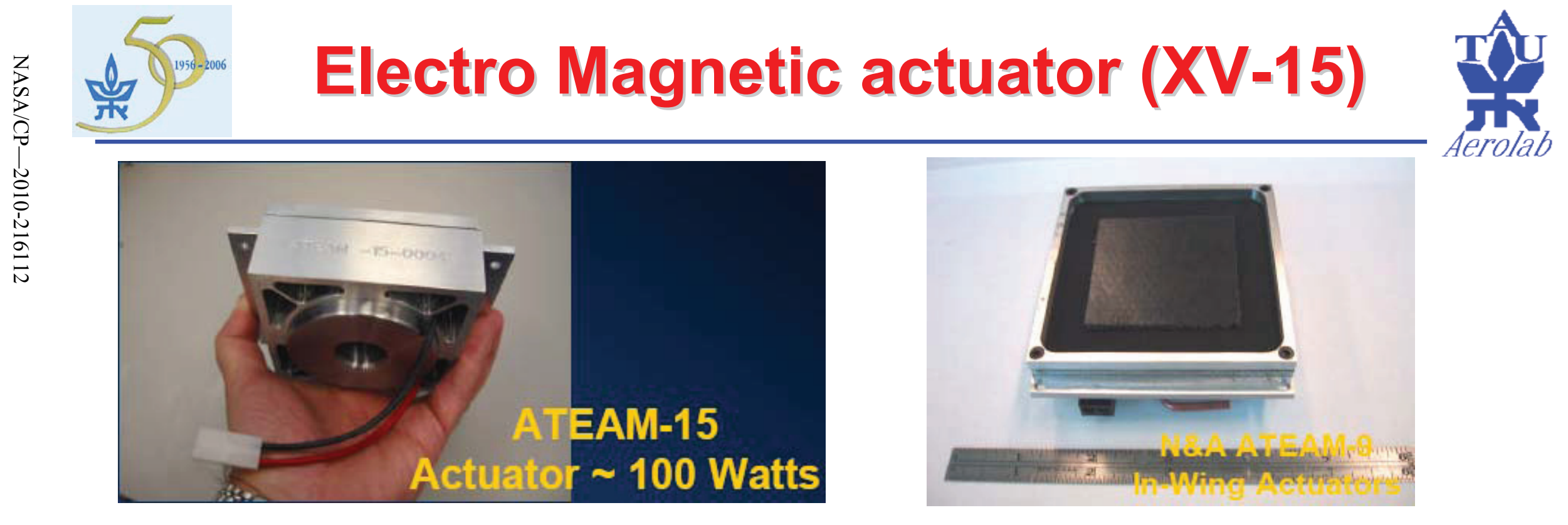

崖

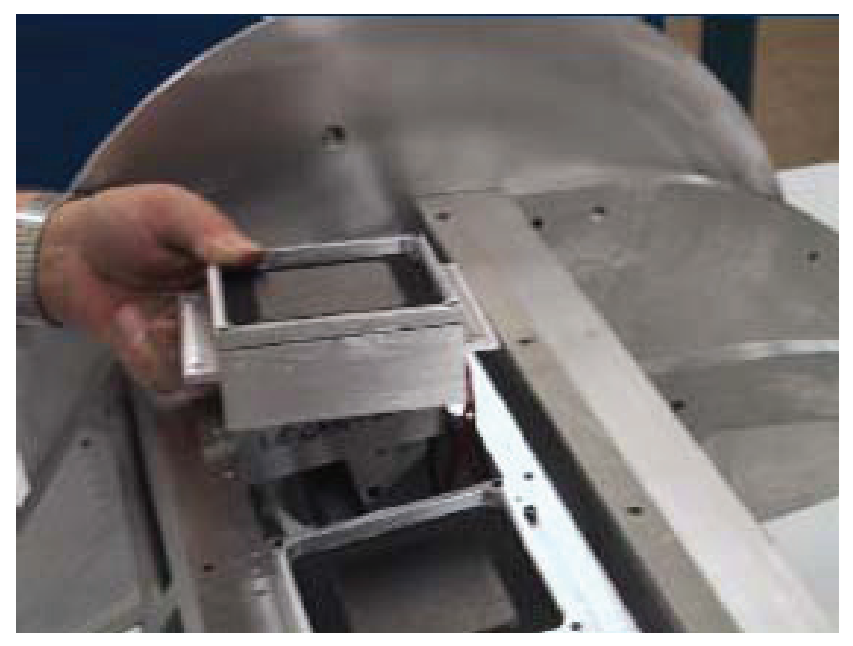

Actu power - 1000W/m $\mathrm{Up}=80 \mathrm{~m} / \mathrm{s}$ Wa $>3 \mathrm{~kg}$

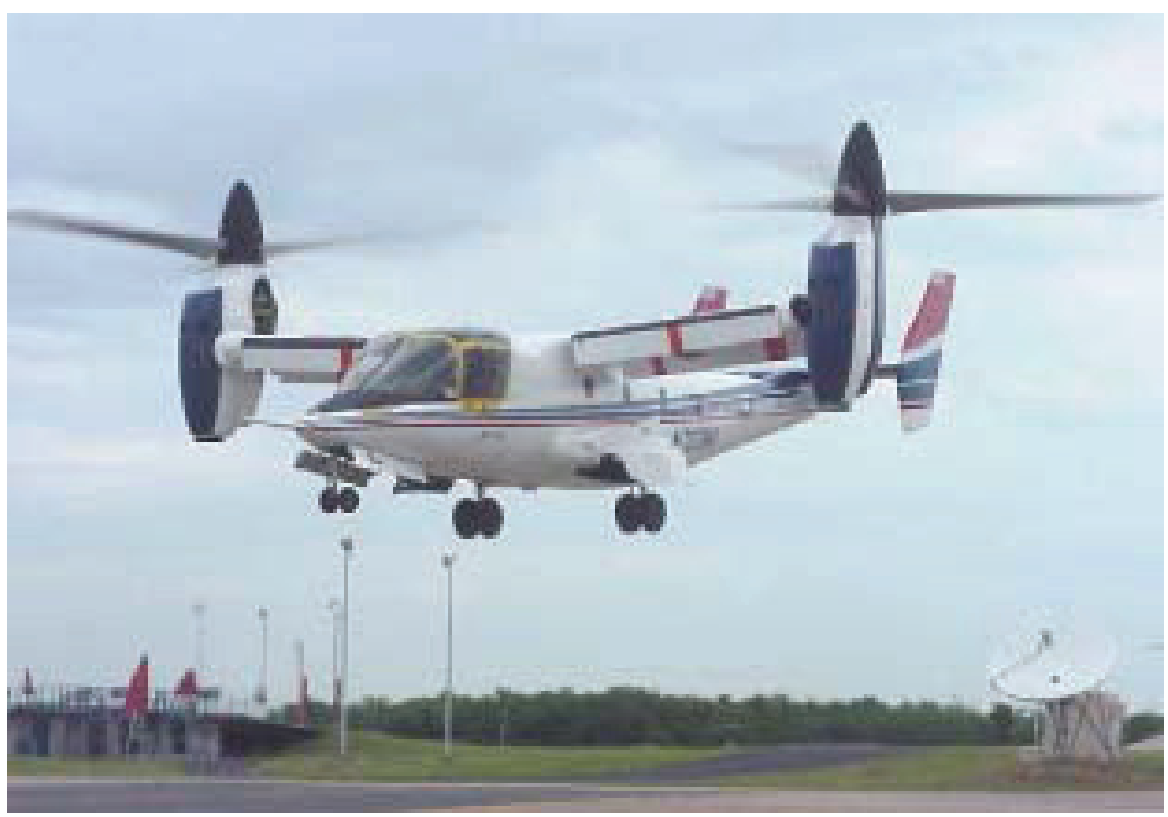



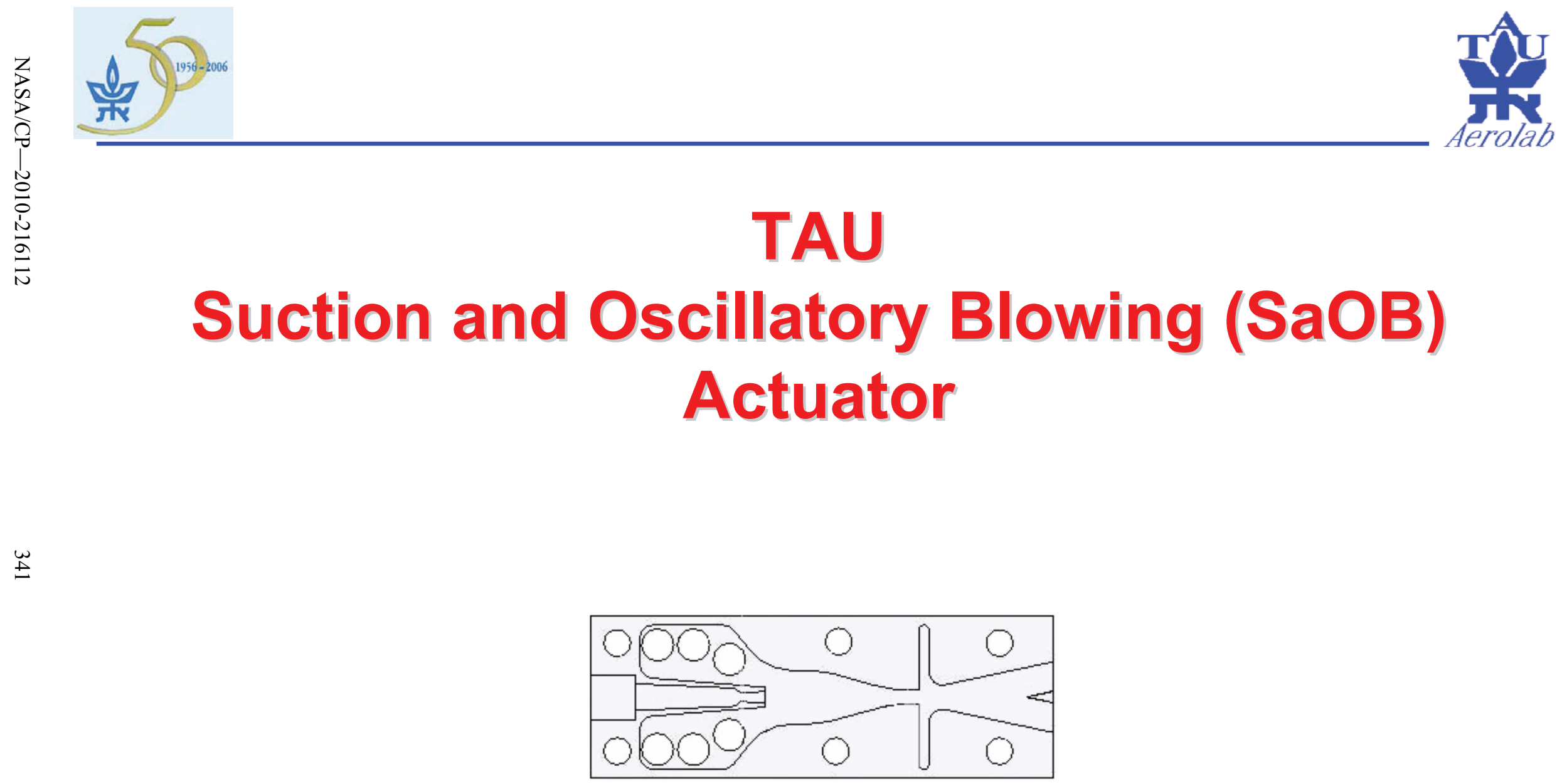


\section{Background}

- Boundary layer separation control by steady suction (note date...)

- Steady, wall tangential blowing

- Oscillatory blowing (directed ZMF)

- The importance of flow instability for efficient AFC

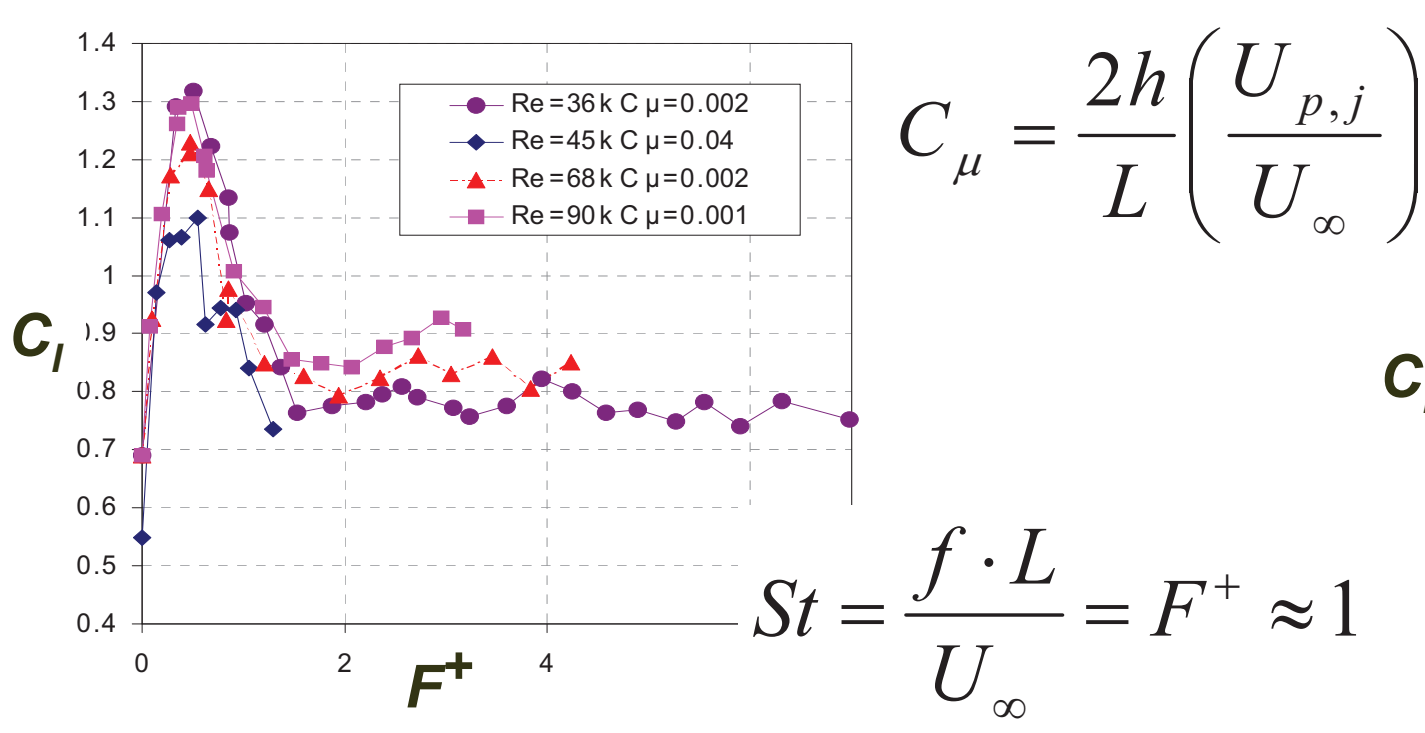

(Yom-Tov \& Seifert, '05)
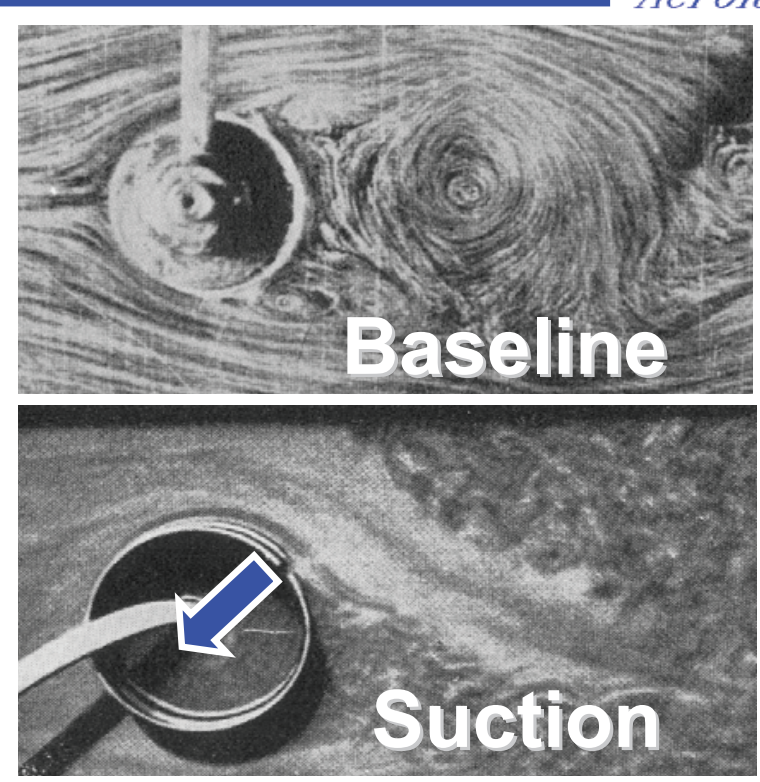

(Prandtl, 1904)

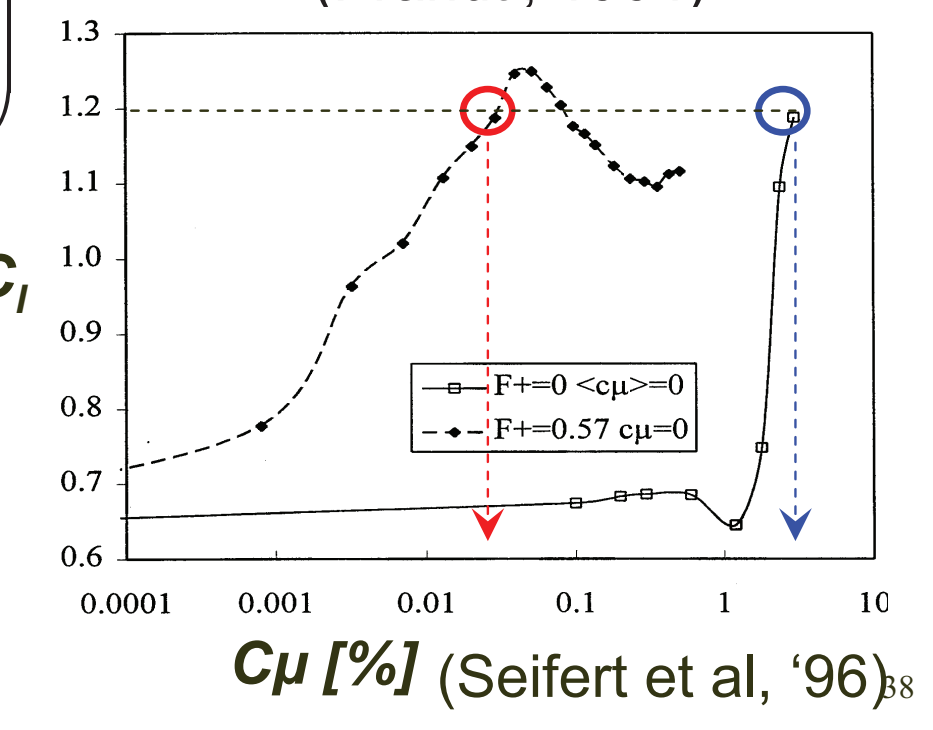




\section{The SaOB actuator concept}

- G. Arwatz (MSc), I. Fono, London MEMS IUTAM, Sep '06

- Available ZNMF devices limited to $M=0.3$

- Suction and oscillatory blowing - high potential for efficient AFC

- Combination of steady suction and oscillatory blowing in one device

- High velocity, near sonic, output

- Wide range of output frequencies

- Enables 3D excitation

- Frequency proportional to flow rat

- No moving parts

- Low power consumption

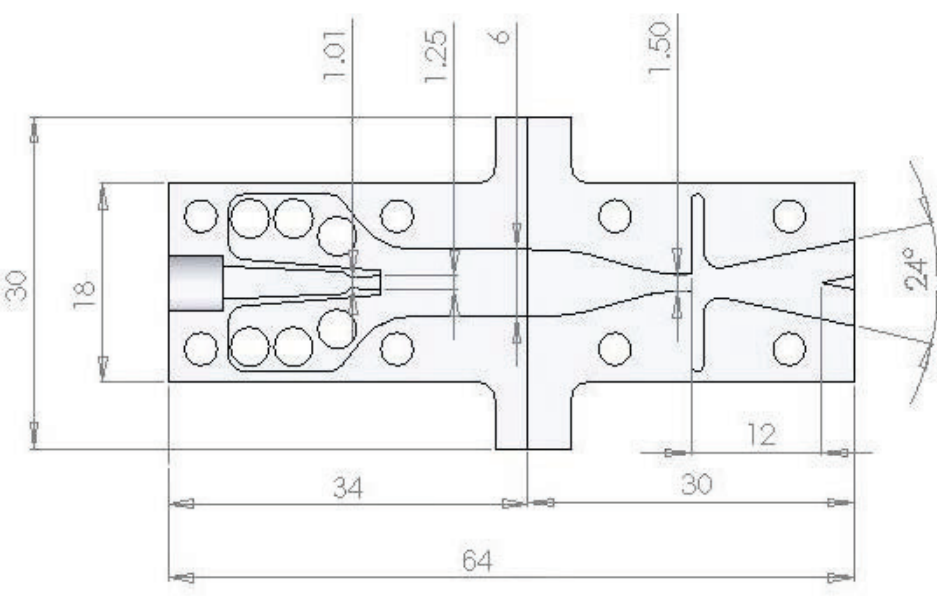




\section{The SaOB actuator concept}

- Combination of ejector and switching valve

\author{
Ejector \\ (steady suction)
}

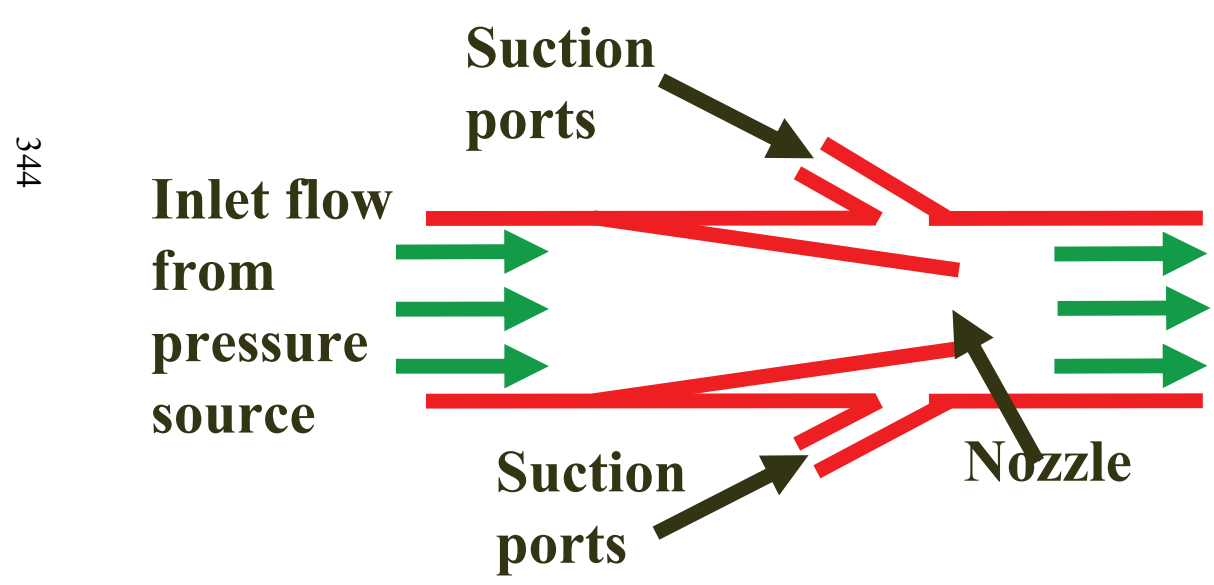

Bi-stable fluidic amplifier (oscillatory blowing)

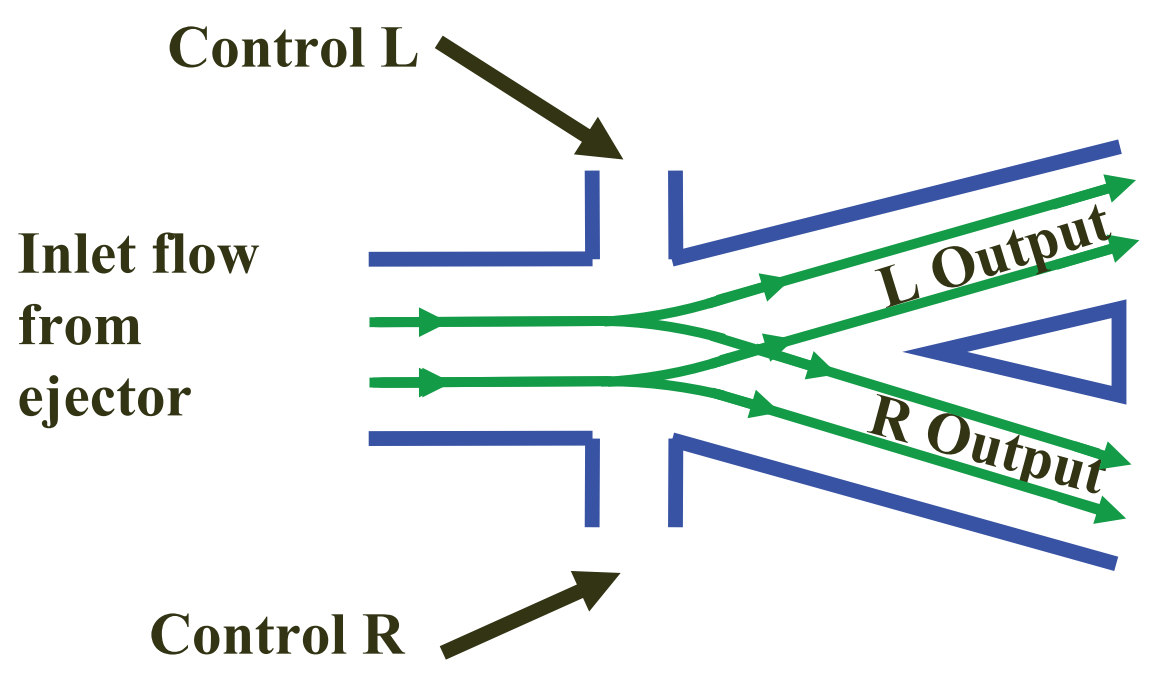




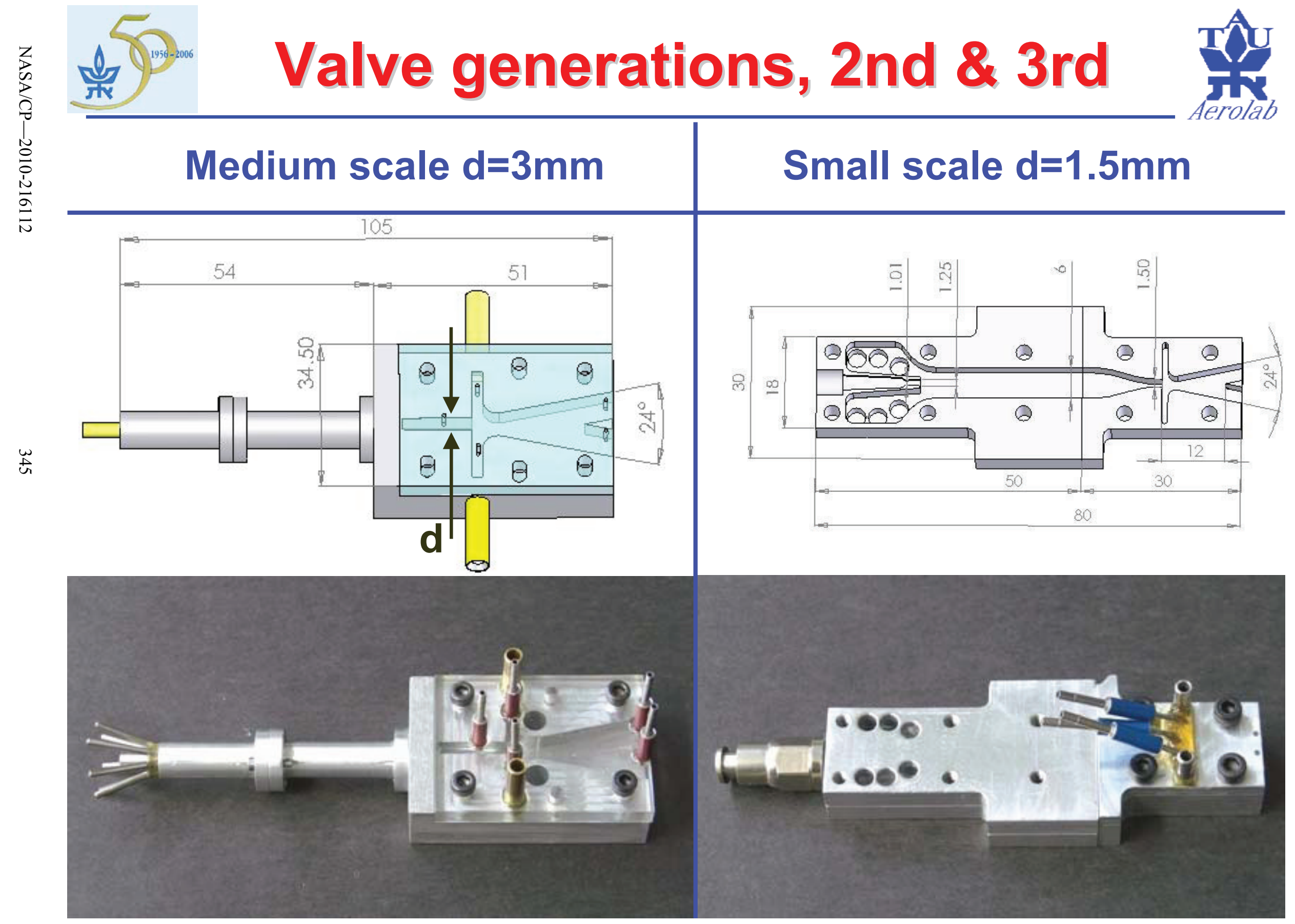




\section{Actuators' Efficiency}

- Overall Figure of Merit

$$
O F M=\frac{F_{a}^{2} U_{p}}{W_{a} P_{a}}
$$

- Still air operation

- SaOB weight - 15 gm

- Thrust-suction+blowing

- Note: OFM Log scale

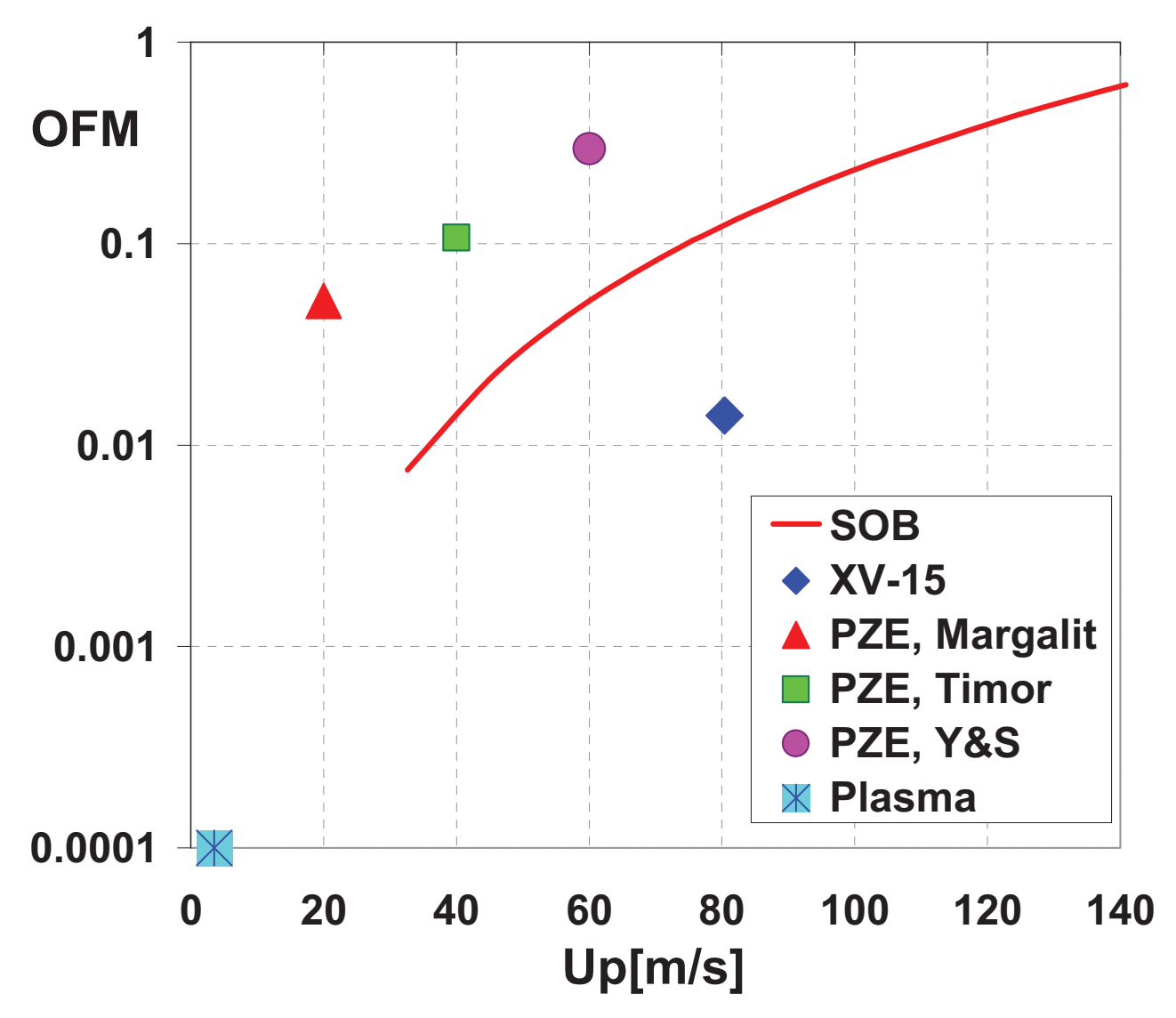




\section{Summary (Actuators)}

- Actuator comparison criteria suggested

- Overall and Aerodynamic figures of merit

- New actuator for steady suction and oscillatory blowing was developed, modeled and characterized

- OFM for ZNMF, Plasma and SaOB actuators compared

- Rare to find data for AFM (Need: Weight, power, Up, L, D)

- Please measure and publish 